\title{
Distillation at high momentum
}

\author{
Colin Egerer, ${ }^{1}$ Robert G. Edwards $\odot,{ }^{2}$ Kostas Orginos, ${ }^{1,2}$ and David G. Richards ${ }^{2}$ \\ (On behalf of the HadStruc Collaboration) \\ ${ }^{1}$ Physics Department, William and Mary, Williamsburg, Virginia 23187, USA \\ ${ }^{2}$ Thomas Jefferson National Accelerator Facility, Newport News, Virginia 23606, USA
}

(Received 25 September 2020; accepted 5 January 2021; published 3 February 2021)

\begin{abstract}
Extraction of hadronic observables at finite momenta from lattice QCD (LQCD) is constrained by the well-known signal-to-noise problems afflicting all such LQCD calculations. Traditional quark smearing algorithms are commonly used tools to improve the statistical quality of hadronic $n$-point functions, provided operator momenta are small. The momentum smearing algorithm of Bali et al. extends the range of momenta that are cleanly accessible and has facilitated countless novel lattice calculations. Momentum smearing has, however, not been explicitly demonstrated within the framework of distillation. In this work we extend the momentum-smearing idea by exploring a few modifications to the distillation framework. Together with enhanced time slice sampling and expanded operator bases engendered by distillation, we find ground-state nucleon energies can be extracted reliably for $|\vec{p}| \lesssim 3 \mathrm{GeV}$ and matrix elements featuring a large momentum dependence can be resolved.
\end{abstract}

DOI: 10.1103/PhysRevD.103.034502

\section{INTRODUCTION}

Lattice field theory is now a thoroughly well-established scheme to quantitatively study strongly interacting theories, such as quantum chromodynamics (QCD), from first principles. With the exception of the lightest pseudoscalar mesons at rest, lattice QCD (LQCD) calculations of the spectrum and properties of hadrons are afflicted by exponentially worsening signal-to-noise ratios as the Euclidean time extent between operators grows. It is thus a key demand of lattice calculations that the hadron of interest saturate correlation functions at as short a Euclidean time separation as possible. Key to satisfying this demand is identifying an operator whose overlap with the hadron of interest is maximized relative to those with other states: $\langle 0|\hat{\mathcal{O}}(\vec{p})| h(\vec{p})\rangle \gg\left\langle 0|\hat{\mathcal{O}}(\vec{p})| h^{\prime}(\vec{p})\right\rangle$.

The most widely used means of accomplishing this is through quark spatial smearing schemes, such as Wuppertal [1] or Jacobi [2] smearing, which act as low-energy filters of hadronic correlation functions, leading to a more rapid relaxation to low-energy eigenmodes. It is thus standard practice to compute hadronic observables where at least one interpolating operator of an $\mathrm{N}$-point function possesses

Published by the American Physical Society under the terms of the Creative Commons Attribution 4.0 International license. Further distribution of this work must maintain attribution to the author(s) and the published article's title, journal citation, and DOI. Funded by SCOAP ${ }^{3}$. a nontrivial spatial extent. However, as pointed out in Ref. [3], spatial smearing of hadronic operators is less than optimal and even detrimental for all but interpolators projected to zero momentum. The authors proposed a remedy, now known as momentum smearing, that involves the introduction of appropriately tuned phase factors onto the underlying gauge links, prior to the subsequent spatial smearing of the quark fields. In effect, a tunable momentum space distribution is constructed by creating an oscillatory spatial profile. The remarkable effectiveness of this procedure was established in [3], wherein the pion and nucleon energies were reliably extracted up to $\sim 2 \mathrm{GeV}$ and $\sim 3 \mathrm{GeV}$, respectively, and the dispersion relations reasonably satisfied.

This robust momentum-smearing technique is now ubiquitous in lattice studies that demand a wide range of momenta, such as the mapping of nucleon electromagnetic form factors (FFs) [4], generalized FFs [5], and semileptonic decay FFs needed to quantify elements of the Cabibbo-Kobayashi-Maskawa matrix [6,7]. Perhaps the greatest usage has been seen in LQCD calculations of matrix elements of certain nonlocal spacelike-separated operators, which when computed over a range of momenta can be related to various light-cone distributions fundamental to hadron structure. Such matrix elements, analyzed in the context of large momentum effective field theory (LaMET) [8,9], have proven useful in understanding the (un)polarized partonic content of the pion and nucleon [10-16] and have yielded encouraging insight into the 
(un)polarized generalized partonic content of the proton [17]. Quark bilinears can be related via coordinate space factorization schemes to light cone distribution amplitudes [18-20], and to quark [21-24] and gluon [25] parton distribution functions (PDFs) within the pseudo-PDF framework [26]. The "lattice cross sections" approach $[27,28]$ generalizes this paradigm to spatially separated gauge-invariant current-current matrix elements, recently employed in $[23,29]$ to determine the valence quark content of the pion.

Although momentum smearing, in concert with Wuppertal or Jacobi smearing, does indeed enhance the overlap of the interpolating operators onto the lowest lying states in the spectrum, there are additional challenges that it does little to ameliorate. First, energy eigenstates contributing to a correlator become dense as the spatial momentum of the correlators increases. Second, the reduced lattice symmetries for correlators at nonzero spatial momentum, together with the contribution of two- and higher-particle states, further increase the density of the higher energies. Distillation [30], when employed with an extended basis of operators that it facilitates, provides a powerful means of addressing these issues, as well as permitting a better sampling of a gauge configuration through explicit momentum projections performed at both source and sink in a two-point correlation function. The use of the variational method within a given lattice symmetry channel, using an extended basis of operators implemented through distillation, has proven essential in mapping the low-lying baryon spectrum of QCD [31,32] and exotic hadrons [33-36], as well as exploring the glueball content in the isoscalar sector of QCD [37]. Recently, the power of this approach has been demonstrated in the calculation of the various nucleon isovector charges [38]. Calculational programs employing distillation have generically limited the spatial momenta to within the shell $\left|a_{s} \vec{p}\right|^{2} \lesssim 4\left(2 \pi / L_{s}\right)^{2}$, where $a_{s}$ is the spatial lattice spacing and $L_{s}$ is the number of time slices in the spatial directions. Here the resultant correlation functions have sufficient momentum-space overlap that the distillation framework does not necessitate modifications. The goal of this work is to supplement distillation with a realization of momentum smearing, thereby increasing the range of hadron momenta accessible, and in so doing demonstrating the efficacy of this approach both for the nucleon energies at higher spatial momenta and for the nucleon charges derived at these high momenta.

The remainder of this paper is organized as follows. We proceed in Sec. II with a brief summary of the distillation framework, and the modifications needed to incorporate momentum smearing within that framework. In Sec. III, we describe its computational implementation, then proceed to a comparison of the nucleon energies with and without momentum smearing on a lattice at the larger of our two pion masses, and identify an optimal procedure for its implementation. In Sec. IV, we extend the investigation to a lighter pion mass, and in particular highlight the efficacy of this approach by determining the renormalized isovector charges of the nucleon in both stationary and boosted frames, with and without the momentum-smearing modifications. In Sec. V we discuss our results for the resultant matrix elements, and their interpretation in terms of both the expected discretization effects and the possible excitedto-ground-state transitions. Concluding remarks are given in Sec. VI.

\section{DISTILLATION}

Distillation [30] is a low-rank approximation to a gaugecovariant smearing kernel, conventionally taken to be the Jacobi smearing kernel $J_{\sigma, n_{\sigma}}(t)=\left(1+\frac{\sigma \nabla^{2}(t)}{n_{\sigma}}\right)^{n_{\sigma}}$ [2]. The tunable parameters $\left\{\sigma, n_{\sigma}\right\}$ allow for variable source "widths" and applications, respectively, such that in the large iteration limit, the kernel approaches that of a spherically symmetric Gaussian. The low-rank approximation is formed by isolating eigenvectors of the discretized three-dimensional gauge-covariant Laplacian

$$
-\nabla^{2}(t) \xi^{(k)}(t)=\lambda^{(k)}(t) \xi^{(k)}(t)
$$

and ordering solutions according to the eigenvalue magnitudes $\lambda^{k}(t)$. The outer product of equal-time eigenvectors defines the distillation smearing kernel

$$
\square(\vec{x}, \vec{y} ; t)_{a b}=\sum_{k=1}^{R_{\mathcal{D}}} \xi_{a}^{(k)}(\vec{x}, t) \xi_{b}^{(k) \dagger}(\vec{y}, t),
$$

where $R_{\mathcal{D}}$ is the chosen rank of the distillation space and color indices $a, b$ are made explicit. Correlation functions formed by Wick-contracting quark fields smeared via (1) can be factorized into distinct reusable components, the elementals and the perambulators. The elementals

$\Phi_{\alpha \beta \gamma}^{(i, j, k)}(t)=\epsilon^{a b c}\left(\mathcal{D}_{1} \xi^{(i)}\right)^{a}\left(\mathcal{D}_{2} \xi^{(j)}\right)^{b}\left(\mathcal{D}_{3} \xi^{(k)}\right)^{c}(t) S_{\alpha \beta \gamma}$,

shown here for the case of baryons, encode the operator construction, where $\mathcal{D}_{i}$ are covariant derivatives and $S_{\alpha \beta \gamma}$ are subduction coefficients encoding how an interpolator with Dirac indices $\{\alpha, \beta, \gamma\}$ constructed in the continuum will mix across irreducible representations (irreps) of a hypercubic lattice and its associated little groups. The perambulators

$$
\tau_{\alpha \beta}^{(l, k)}\left(t^{\prime}, t\right)=\xi^{(l) \dagger}\left(t^{\prime}\right) M_{\alpha \beta}^{-1}\left(t^{\prime}, t\right) \xi^{(k)}(t)
$$

encode the propagation of the quarks between elements of the distillation space, where $M$ is the Dirac operator. It is this factorization of the quark propagation from the construction of the interpolating operators that enables the 
computationally efficient implementation of the variational method with an extended basis of operators.

\section{A. Momentum smeared distillation}

Distillation is quite costly initially in both computational storage and the construction of its components. Moreover, the rank $R_{\mathcal{D}}$ is expected to scale with the lattice spatial volume in order to maintain the same resolution in correlation functions on different ensembles [30]. This is particularly significant for the construction of the correlation functions, where the needed Wick contractions for meson and baryon two-point functions scale as $R_{\mathcal{D}}^{3}$ and $R_{\mathcal{D}}^{4}$, respectively. Thus an implementation of momentum smearing within distillation must seek to minimize the number of additional distillation vectors included in the basis, and in particular avoid the use of a distinct eigenvector basis for each momentum of the correlation functions.

With such a scenario in mind, one might consider modifying a set of eigenvectors according to

(1) Single Phase

$$
\tilde{\xi}_{a}^{(k)}(\vec{z}, t)=e^{i \vec{\xi} \cdot \vec{z}} \xi_{a}^{(k)}(\vec{z}, t)
$$

(2) Opposing Phases

$$
\tilde{\xi}_{a}^{(k)}(\vec{z}, t)=2 \cos (\vec{\zeta} \cdot \vec{z}) \xi_{a}^{(k)}(\vec{z}, t)
$$

(3) Identity and Opposing Phases

$$
\tilde{\xi}_{a}^{(k)}(\vec{z}, t)=[1+2 \cos (\vec{\zeta} \cdot \vec{z})] \xi_{a}^{(k)}(\vec{z}, t)
$$

(4) Multiple Unidirectional Phases

$$
\tilde{\xi}_{a}^{(k)}(\vec{z}, t)=\left[e^{i \vec{\zeta}_{1} \cdot \vec{z}}+e^{i \vec{\zeta}_{2} \cdot \vec{z}}\right]_{\zeta_{1} \neq \zeta_{2}} \xi_{a}^{(k)}(\vec{z}, t),
$$

such that overlaps for several, potentially opposing, hadron momenta could be simultaneously improved. A schematic qualitative picture of these candidate implementations is depicted in Fig. 1.

An important requirement of any modification of distillation is the preservation of translational invariance, since that is essential for the projection to states to definite momentum. It is straightforward to show that the perambulators with the Type-1 modification are indeed invariant under the translation of the phase through $\vec{x} \rightarrow \vec{x}+\vec{d}$ :

$$
\begin{aligned}
\tilde{\tau}_{\mu \nu}^{i j}\left(t^{\prime}, t\right)= & \xi^{(i) \dagger}\left(\vec{x}, t^{\prime}\right) e^{-i \vec{\xi} \cdot(\vec{x}+\vec{d})} M_{\mu \nu}^{-1}\left(\vec{x}, t^{\prime} ; \vec{y}, t\right) \\
& \times e^{i \vec{\xi} \cdot(\vec{y}+\vec{d})} \xi^{(j)}(\vec{y}, t) \\
= & \xi^{(i) \dagger}\left(\vec{x}, t^{\prime}\right) e^{-i \vec{\xi} \cdot \vec{x}} M_{\mu \nu}^{-1}\left(\vec{x}, t^{\prime} ; \vec{y}, t\right) e^{i \vec{\xi} \cdot \vec{y}} \xi^{(j)}(\vec{y}, t) .
\end{aligned}
$$

Such translation invariance fails for the other implementations of momentum smearing, as we show below for phasing of Type 4:

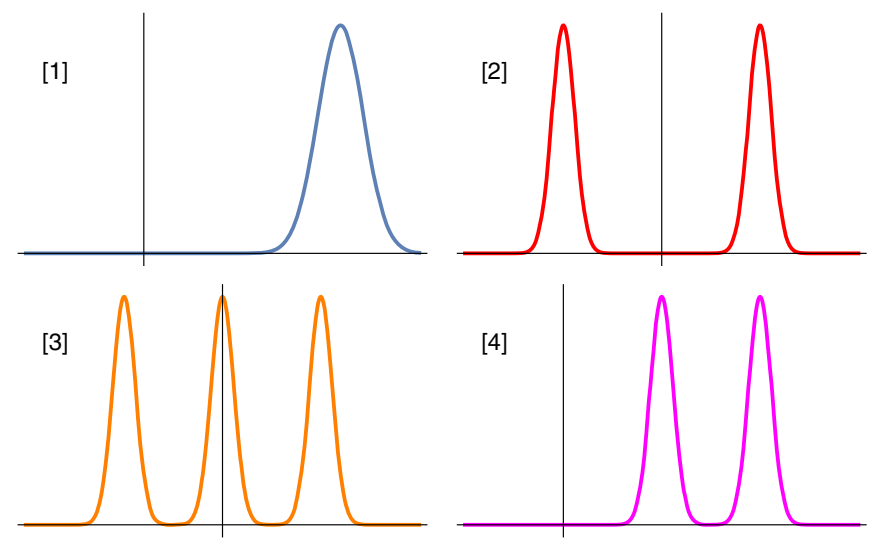

FIG. 1. Qualitative momentum space overlaps following modification of a computed eigenvector basis. Panels 2-4 expressly violate translation invariance, but would dramatically reduce computational cost were translational symmetry preserved.

$$
\begin{aligned}
\tilde{\tau}_{\mu \nu}^{i j}\left(t^{\prime}, t\right)= & \xi^{(i) \dagger}\left(\vec{x}, t^{\prime}\right)\left\{e^{-i \vec{\zeta}_{2} \cdot(\vec{x}+\vec{d})}+e^{-i \overrightarrow{\zeta_{1}} \cdot(\vec{x}+\vec{d})}\right\} \\
& \times M_{\mu \nu}^{-1}\left(\vec{x}, t^{\prime} ; \vec{y}, t\right)\left\{e^{i \vec{\zeta}_{1} \cdot(\vec{y}+\vec{d})}+e^{i \overrightarrow{\zeta_{2}} \cdot(\vec{y}+\vec{d})}\right\} \xi^{(j)}(\vec{y}, t) \\
= & \xi^{(i) \dagger}\left(\vec{x}, t^{\prime}\right) e^{-i \vec{\zeta}_{2} \cdot \vec{x}} e^{i\left(\vec{\zeta}_{1}-\vec{\zeta}_{2}\right) \cdot \vec{d}} M_{\mu \nu}^{-1}\left(\vec{x}, t^{\prime} ; \vec{y}, t\right) \\
& \times e^{i \vec{\zeta}_{1} \cdot \vec{y}} \xi^{(j)}(\vec{y}, t)+\left\{\vec{\zeta}_{1} \leftrightarrow \vec{\zeta}_{2}\right\}+\mathcal{T} \cdot \mathcal{I} .,
\end{aligned}
$$

where we find a combination of translationally invariant $\left(\mathcal{T}\right.$. I. ) and variant pieces for $\vec{\zeta}_{1} \neq \vec{\zeta}_{2}$. Thus in the remainder of this paper, we consider only the phasing of Type 1 , and refer to the modified eigenvector basis as "phased."

The momentum smearing scheme of Ref. [3] reweights gauge fields $U_{\mu}[x]$ in a boost direction $z_{\mu}$ with weight $\zeta=\frac{2 \pi}{L} \mathfrak{r}$ according to

$$
\tilde{U}_{\mu}[x]=e^{i \frac{2 \pi}{L} \mathfrak{z} z_{\mu}} U_{\mu}[x]
$$

prior to quark source creation, where $\mathfrak{r} \in \mathbb{R}$. As phases are applied to the underlying gauge configurations prior to determination of the eigenvectors, the configurations can safely be smeared with unallowed lattice momenta as highlighted in [3]. Thus it is sufficient to modify the previously computed eigenvectors, limiting phases to allowed lattice momenta. In particular we consider the phase factors

$$
\begin{gathered}
\vec{\zeta}=\frac{2 \pi}{L} \hat{z}, \\
\vec{\zeta}=2 \cdot \frac{2 \pi}{L} \hat{z},
\end{gathered}
$$

corresponding to one and two units of the allowed lattice momenta. We remark that phases applied in the $-\hat{z}-$ direction improve momentum space overlaps for $a p_{z}<0$ but are not presented herein for brevity. 


\section{DEMONSTRATION OF EFFICACY}

We employ two isotropic clover ensembles, with $2 \oplus 1$ flavors, of extent $32^{3} \times 64$, an inverse coupling $\beta=6.3$, corresponding to a lattice spacing $a \simeq 0.094 \mathrm{fm}$, and with pion masses of 358 and $278 \mathrm{MeV}$, respectively. These are cataloged in Table I; further details of the ensembles are contained in Refs. [39,40]. To first establish the feasibility of our candidate implementation, we employ the ensemble at the heavier pion mass, herein denoted by $a 094 \mathrm{~m} 358$. The figure of merit we use is to extract the ground-state nucleon dispersion relation for as large a range of momentum as possible.

Calculations were performed for four distinct (randomized) source temporal origins on 100 configurations of the $a 094 m 358$ ensemble, with each configuration separated by $10 \mathrm{HMC}$ trajectories; this small number of configurations was found sufficient to quantitatively demonstrate the effectiveness of distillation for the nucleon energies and dispersion relation. We employed $R_{\mathcal{D}}=64$ eigenvectors, where the gauge fields in the Laplacian were smoothed via ten iterations of stout smearing [41] with smearing parameter $\rho_{i j}=0.08$ and $\rho_{\mu 4}=\rho_{4 \mu}=0$. We remark the choice of $R_{\mathcal{D}}$ is driven by a balance between control of excited states and numerical cost to construct/contract the various distillation components. We justify our choice of $R_{\mathcal{D}}=64$ in the Appendix.

\section{A. Interpolator construction}

The regularization of QCD through lattice discretization explicitly breaks continuum rotational symmetry, and consequently baryons at rest are now cataloged according to the double-cover irreps of the octahedral group $O_{h}^{D}$. Thus mass eigenstates once cataloged by $J^{P}$ must now be isolated according to their patterns of subduction across the finite number of irreps $\Lambda$ of $O_{h}^{D}$. The construction of the nucleon operators follows the procedure introduced in Refs. [31,32], which we summarize now, and are expressed in terms of the baryon elementals introduced in Eq. (2). These operators are projections onto the lattice irreps of discretized continuumlike operators, which we classify according to the spectroscopic notation $N^{(2 S+1)} L_{\mathcal{P}} J^{P}$, where $S$ represents the Dirac spin, $L$ the angular momentum introduced via derivatives, $\mathcal{P}$ the permutational symmetry of such derivatives, and $J^{P}$ the total angular momentum and parity of the nucleon interpolator $N$.

TABLE I. Lattice ensembles utilized throughout this work. The number of distillation eigenvectors $R_{\mathcal{D}}$ and distinct source positions $N_{\text {srcs }}$ per configuration are also indicated.

\begin{tabular}{lcccccc}
\hline ID & $a[\mathrm{fm}]$ & $m_{\pi}[\mathrm{MeV}]$ & $L^{3} \times N_{t}$ & $N_{\text {cfg }}$ & $N_{\text {srcs }}$ & $R_{\mathcal{D}}$ \\
\hline$a 094 m 358$ & $0.094(1)$ & $358(3)$ & $32^{3} \times 64$ & 100 & 4 & 64 \\
$a 094 m 278$ & $0.094(1)$ & $278(4)$ & $32^{3} \times 64$ & 259 & 4 & 64 \\
\hline \hline
\end{tabular}

To best capture the ground-state $J^{P}=\frac{1}{2}^{+}$nucleon at rest, which trivially subduces into the $G_{1 g}$ irrep of $O_{h}^{D}$, we use a basis of nonrelativistic interpolators [31,32]

$$
\begin{aligned}
& \mathcal{B}_{\vec{p}=0}=\left\{N^{2} S_{S} \frac{1+}{2}, N^{2} S_{M} \frac{1+}{2}, N^{2} S_{S}^{\prime} \frac{1+}{2}, N^{2} P_{A} \frac{1+}{2},\right. \\
& \left.N^{2} P_{M} \frac{1+}{2}, N^{4} P_{M}{ }_{2}^{1+}, N^{4} D_{M} \frac{1+}{2}\right\}
\end{aligned}
$$

that admit a flexible description of the radial/orbital nucleon structure-we note $N^{2} P_{M \frac{1}{2}}{ }^{+}$and $N^{4} P_{M}{ }^{\frac{1}{2}}{ }^{+}$are of hybrid construction.

Projection of the lattice interpolating fields to nonzero spatial momenta $(\vec{p} \neq \overrightarrow{0})$ further breaks the $O_{h}^{D}$ symmetry group to little groups dependent on the $*(\vec{p})$ [42], and furthermore mixes states of different parities. Here we consider only boosts along a spatial axis, which are especially important for PDF calculations in the LaMET and pseudo-PDF frameworks. In this case, the little group is the order-16 dicyclic group or Dic 4 . The framework for the construction of the operators, specialized to the case of mesons, is given in Ref. [43]. The genesis is the classification of operators of definite helicity, and therefore we extend our basis to include those both of higher spins and of negative parity, which are then subduced to the little group. In particular, our basis is extended as follows, based on the study of the nucleon spectrum and the dominant operators in Ref. [32] ${ }^{1}$ :

$$
\begin{aligned}
& \mathcal{B}_{\vec{p} \neq 0}=\left\{N^{2} S_{S} \frac{1{ }^{+}}{2}, N^{2} S_{M}{ }^{\frac{1}{2}}, N^{2} P_{A} \frac{1+}{2}, N^{2} P_{M}{ }^{\frac{1}{2}},\right.
\end{aligned}
$$

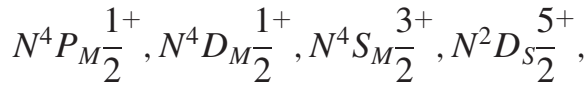

$$
\begin{aligned}
& N^{2} P_{M} \frac{1-}{2}, N^{4} P_{M} \frac{1-}{2}, N^{2} P_{M} \frac{3^{-}}{2}, N^{4} P_{M} \frac{3^{-}}{2},
\end{aligned}
$$

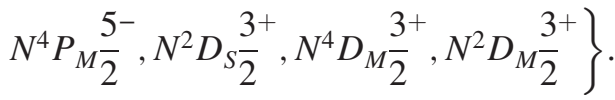

We emphasize that the density of the (discrete) energy spectrum for the nucleon is expected to be considerably greater for states in motion compared with those at rest for the following reasons. First, as the spatial momentum is increased, the separation between the energies of a given state is compressed. Second, through the reduced symmetries, even in the continuum that enables more states to contribute within a given symmetry channel.

\section{B. Variational analysis}

The factorization of a correlation function intrinsic to distillation facilitates the use of an extended basis of interpolators at source and sink, without recomputation

\footnotetext{
${ }^{1}$ Note $N^{2} S_{S 2}^{\prime} \frac{1}{}{ }^{+}$is removed from our interpolator basis.
} 


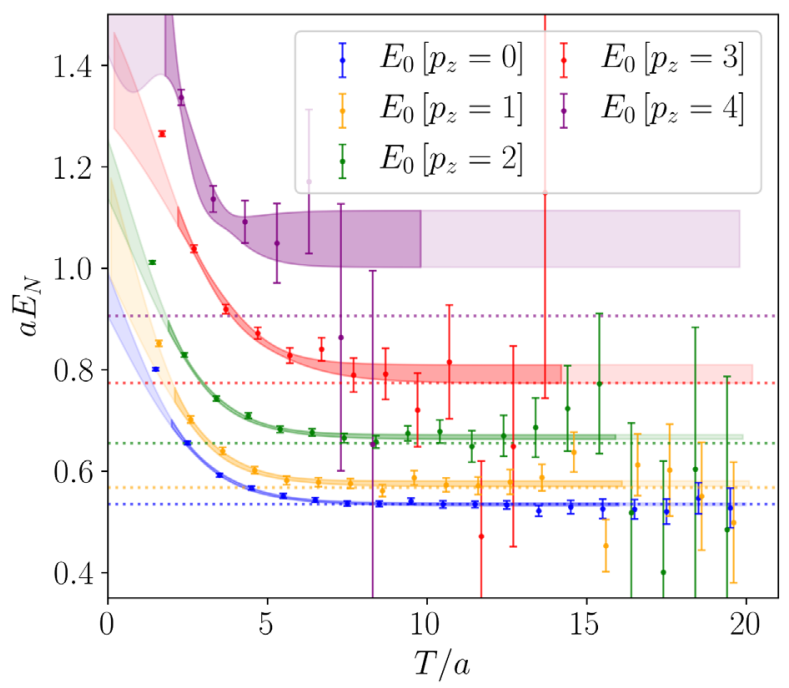

(a)

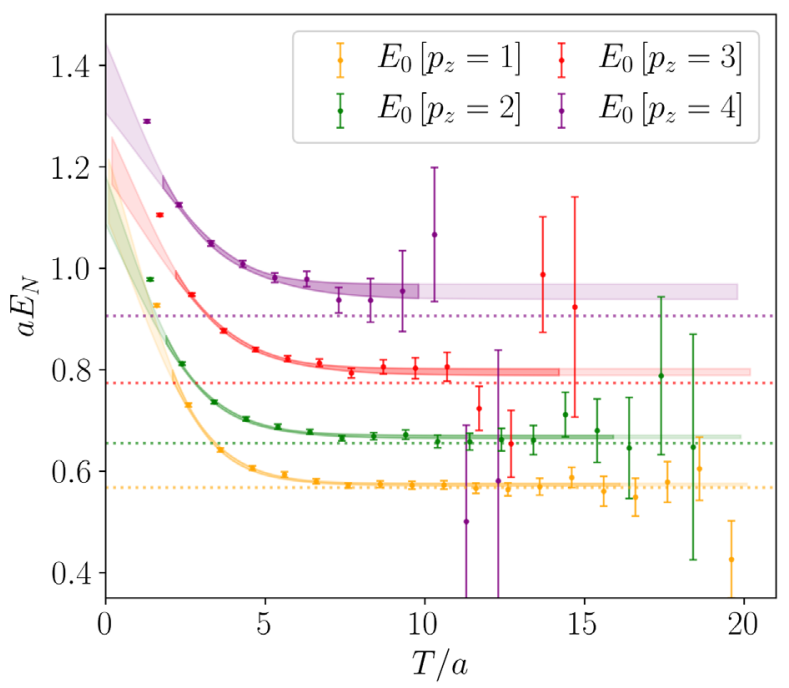

(b)

FIG. 2. (a) The left-hand and (b) right-hand plots show the effective energies for the nucleon, obtained on the $a 094 m 358$ ensemble, using a single, local interpolating operator $N^{2} S_{S_{2}}{ }^{+}$, subduced to the relevant little group, constructed with (a) unphased and (b) phased distillation eigenvectors, respectively. Data are shown for points where the signal-to-noise ratios are (a) $\geq 1.35$ and (b) $\geq 2$, and are shifted for legibility. The bands show the two-state fits to the correlators, as described in the text, where the dark region indicates data included in the fits. The dashed lines represent the energies expected from the continuum dispersion relation using the nucleon mass obtained from the fit to the $\vec{p}=0$ correlator.

of quark propagators as in standard smearing schemes. We are then able to perform a variational analysis in the nucleon $G_{1 g}$ channel at rest [Eq. (7)] and for all boosted frames in the $\mathrm{Dic}_{4}$ little group [Eq. (8)]. We start with a matrix of correlation functions

$$
C_{i j}(T, \vec{p})=\left\langle 0\left|\mathcal{O}_{i}(T,-\vec{p}) \mathcal{O}_{j}^{\dagger}(0, \vec{p})\right| 0\right\rangle,
$$

where $\vec{p}$ is the momentum projection and $\mathcal{O}^{\dagger}$ is selected from some interpolator basis $\mathcal{B}$; we reiterate that distillation enables momentum projections at both source and sink time slices, respectively. The variational method corresponds to the solution of a generalized eigenvalue problem (GEVP) of the form

$$
C(T, \vec{p}) v_{\mathbf{n}}\left(T, T_{0}\right)=\lambda_{\mathbf{n}}\left(T, T_{0}\right) C\left(T_{0}, \vec{p}\right) v_{\mathbf{n}}\left(T, T_{0}\right) .
$$

Optimal operators, in the variational sense, for the energy eigenstates $|\mathbf{n}\rangle$ are defined by $\sum_{i} v_{\mathbf{n}}^{i} \mathcal{O}_{i}^{\dagger}$. Associated with each eigenvector is a principal correlator $\lambda_{\mathbf{n}}\left(T, T_{0}\right)$. We will obtain the energy associated with each state $|\mathbf{n}\rangle$ by fitting its principal correlator according to

$$
\lambda_{\mathbf{n}}\left(T, T_{0}\right)=\left(1-A_{\mathbf{n}}\right) e^{-E_{\mathbf{n}}\left(T-T_{0}\right)}+A_{\mathbf{n}} e^{-E_{\mathbf{n}}^{\prime}\left(T-T_{0}\right)} .
$$

The inclusion of a second exponential serves to quantify the extent to which a principal correlator is dominated by a single state, for which any deviation is encapsulated by the amplitude $A_{\mathbf{n}}$ and "excited" energy $E_{\mathbf{n}}^{\prime}$. Further details, and in particular regarding the selection of $T_{0}$ and the conditions used to enforce orthogonality of eigenvectors $v_{\mathbf{n}}\left(T, T_{0}\right)$, are contained in Refs. [34,38].

\section{Efficacy of phased distillation and nucleon dispersions}

We benchmark the standard distillation implementation, without phasing, by first computing ground-state nucleon energies using the single, local interpolating operator $N^{2} S_{S_{2}}{ }^{1+}$, the analog to standard nucleon interpolators, for $a p_{z} \leq 4(2 \pi / L)$. We fit the two-point functions to the twoexponential form

$$
C_{\mathrm{fit}}^{2 \mathrm{pt}}(T, \vec{p})=e^{-E(\vec{p}) T}\left(a+b e^{-\Delta E T}\right),
$$

where $\Delta E$ is the gap between the ground- and excited-state energies, and priors are introduced to ensure the positivity of the overlap parameters $\{a, b\}$. To avoid possible contact terms arising from the use of the Wilson-clover action, only temporal separations greater than one are included in the fit. The data and the resulting fits are shown in Fig. 2(a). For the lowest momenta $a p_{z} \leq 2(2 \pi / L)$, the data exhibit a clear signal over the large range of $T / a$ and are well described by a two-state fit. Furthermore, the resulting ground-state energies are in excellent agreement with the expectations from the continuum dispersion relation $E^{2}=$ $m^{2}+p^{2}$. However, for momenta $a p_{z}=\{3,4\} \times(2 \pi / L)$, not only does the signal-to-noise ratio degrade rapidly, but a two-state fit becomes insufficient to capture the contributions of excited states to the correlator signal. The latter is 


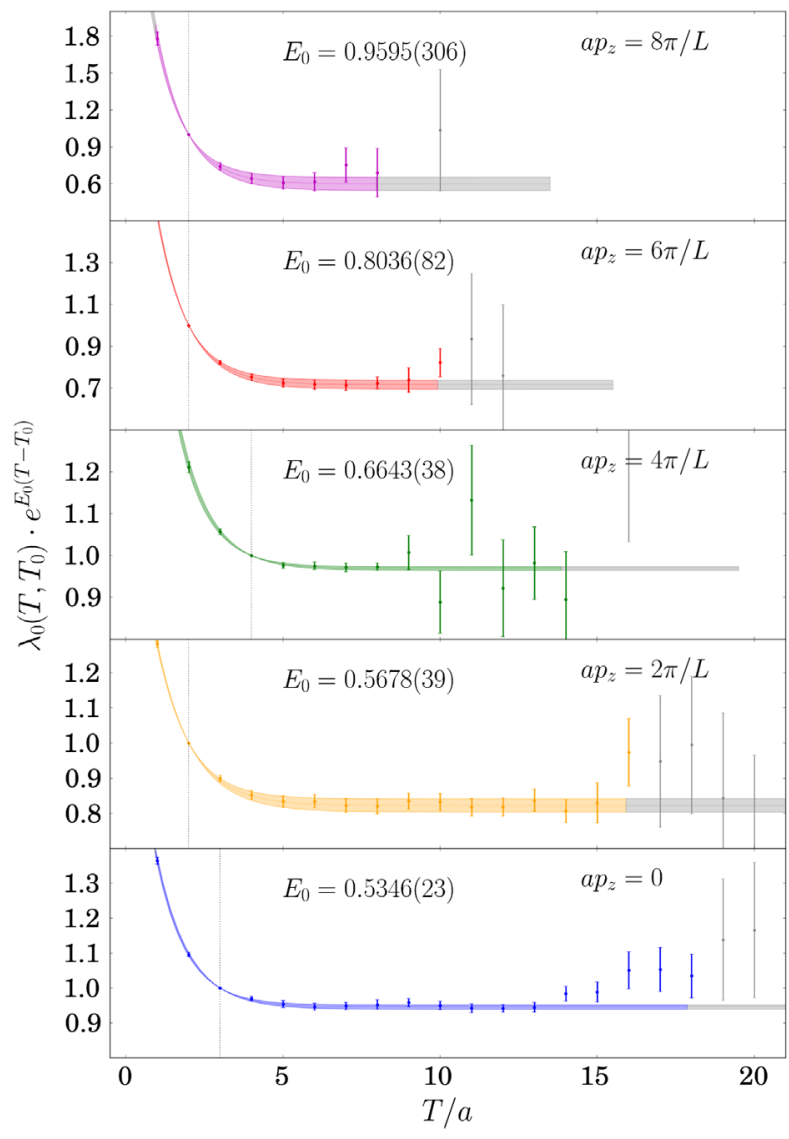

(a)

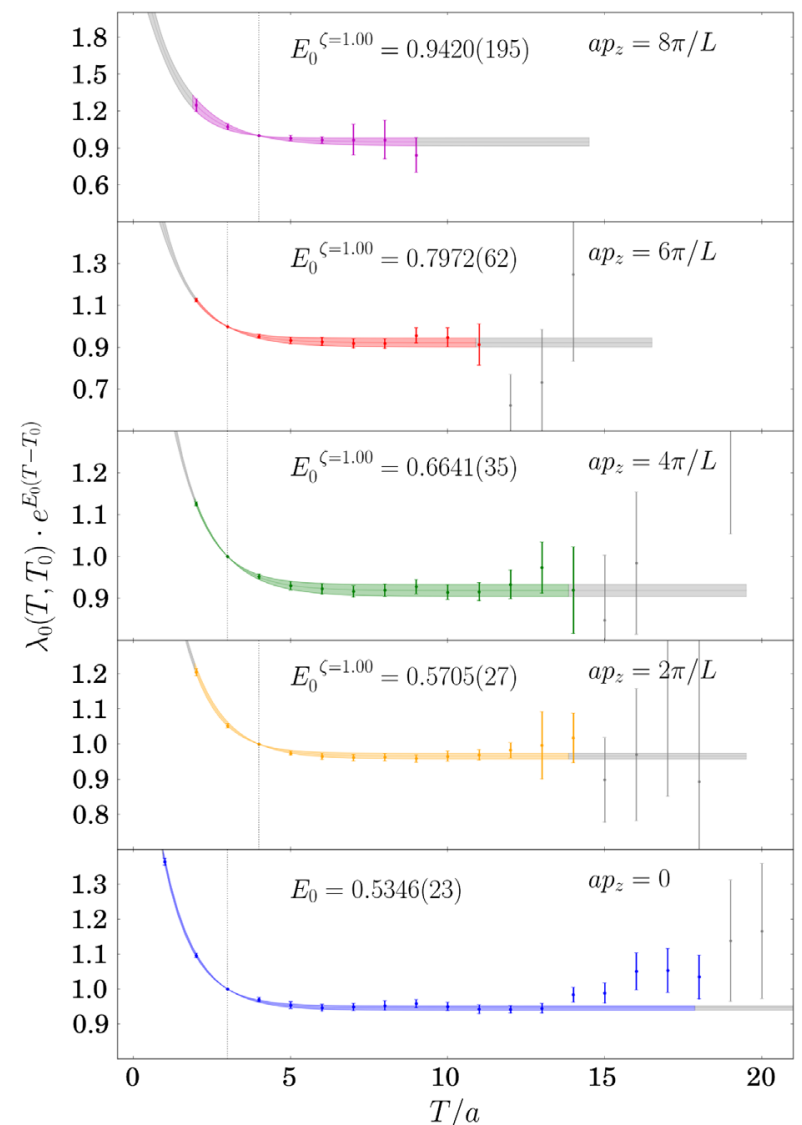

(b)

FIG. 3. The ground-state nucleon principal correlators for the $a 094 m 358$ ensemble using a projected interpolator within each momentum channel obtained from the $\mathcal{B}_{\vec{p} \neq \overrightarrow{0}}$ interpolator basis subduced into the relevant little group. The left-hand and right-hand panels are obtained from the unphased and phased eigenvectors, with one unit of momentum, respectively. The ground-state principal correlator for the unphased $\mathcal{B}_{\vec{p}=0}$ basis is shown for reference (blue). In each case, data are shown for signal-to-noise ratios $\geq 2$. The bands show the two-exponential fits of Eq. (11), with data excluded from the fits in gray. Both the data and fits are shown as $\lambda_{0} e^{E_{0}\left(T-T_{0}\right)}$, where $E_{0}$ is the lowest-lying energy obtained from the fit.

seen by the tension between the fit and correlator for Euclidean separations $T / a \leq 5$. Inclusion of additional states in the functional of (12) would undoubtedly better describe early times in the $a p_{z}=\{3,4\} \times(2 \pi / L)$ signals, but the lack of statistically meaningful signals beyond $T / a \simeq 10$ presents a serious limitation.

Figure 2(b) features the $N^{2} S_{S} \frac{1}{2}$ correlators where the underlying eigenvectors are phased with one unit of momentum, as in Eq. (5). While there is only a modest improvement in the statistical precision of the large- $T / a$ signal for $a p_{z}=\{1,2\} \times(2 \pi / L)$, a dramatic improvement is seen for the $a p_{z}=\{3,4\} \times(2 \pi / L)$ signals. The improved statistical precision with phasing also serves to expose deviations of the energies from the expectations of the continuum dispersion relation. These discrepancies could arise from discretization effects, or from incomplete determination of the ground-state correlation function. It is this latter possibility that we now try to control through the use of the variational method.
We performed the variational analysis on the matrix of correlation functions formed by interpolators in the $\mathcal{B}_{\vec{p}=\overrightarrow{0}}$ [Eq. (7)] and $\mathcal{B}_{\vec{p} \neq 0}$ bases [Eq. (8)]. We first applied the variational method to the unphased basis to determine the improvement this provides with respect to the single operator used above. We then performed the same analyses with distillation spaces modified according to (5) (one unit of momentum) and (6) (two units of momentum), over the momentum ranges $1 \leq(2 \pi / L)^{-1} a p_{z} \leq 4$ and $4 \leq(2 \pi / L)^{-1} a p_{z} \leq 8$, respectively. These momentum ranges were chosen to emphasize that, although one would naively expect eigenvectors modified according to (5) to have optimal overlap with momenta $a p_{z}=3(2 \pi / L)$ and (6) with $a p_{z}=6(2 \pi / L)$, a broad coverage in momentum is possible within each modified space, thereby obviating the need to use many distillation bases each with its own computational cost.

The principal correlators, together with the two-state fits of Eq. (11), are shown in the left- and right-hand plots of 
Fig. 3 for the cases of unphased eigenvectors and phased eigenvectors with one unit momentum, respectively. Compared to the use of phasing with the single $N^{2} S_{S_{2}}{ }^{\frac{1}{+}}$ interpolator, the gains afforded by a variational analysis of the phased operator basis appear less dramatic than the use of an unmodified basis. The principal correlators in each case demonstrate a rather uniform plateau very close to unity, indicative of single eigenstate dominance. However, the phased principal correlators are much better determined and lead to more precise determinations of the ground-state nucleon energies. For example in the $a p_{z}=4(2 \pi / L)$ case, the extracted nucleon energy from the phased principal correlator is $\sim 35 \%$ more precise than the unphased equivalent.

For the highest momenta $4 \leq(2 \pi / L)^{-1} a p_{z} \leq 8$ a comparison with the unphased principal correlators is not possible due to expected statistical fluctuations. We instead show in Fig. 4 principal correlators for $4 \leq(2 \pi / L)^{-1} a p_{z} \leq 8$, where now the eigenvectors are phased with two units of allowed lattice momenta (6). Though the principal

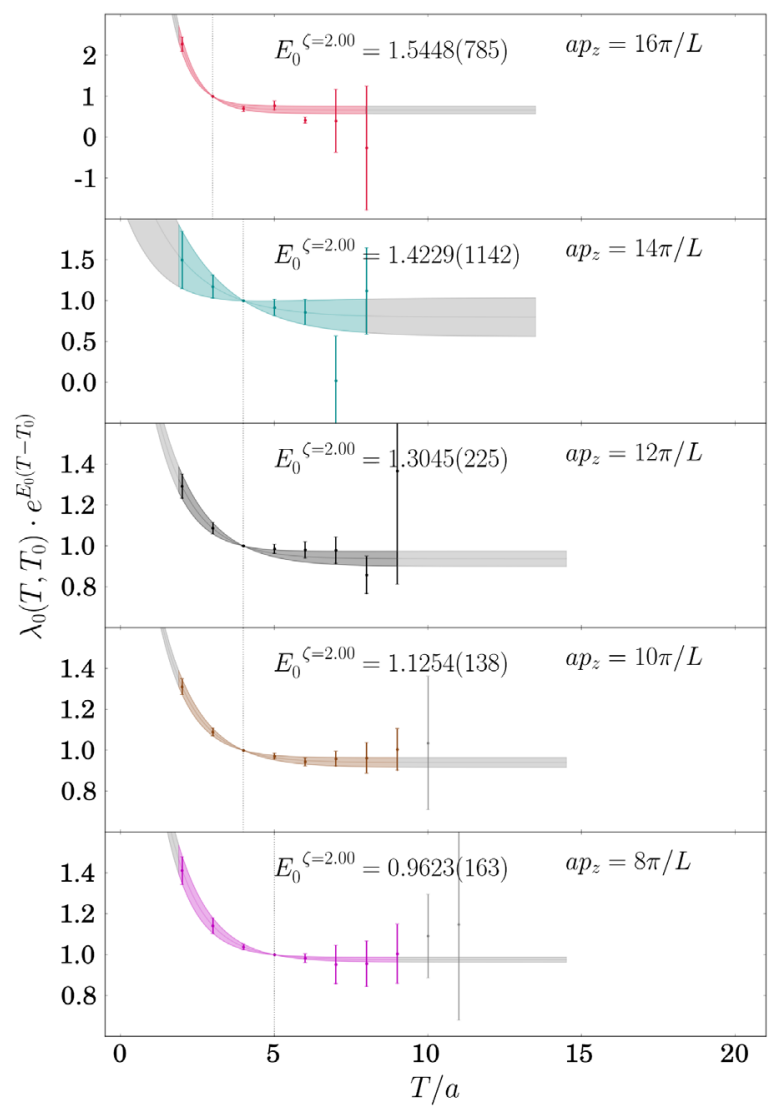

FIG. 4. The ground-state nucleon principal correlators for the $a 094 m 358$ ensemble using a projected interpolator within each momentum channel obtained from the $\mathcal{B}_{\vec{p} \neq 0}$ interpolator basis subduced into the relevant little group. The eigenvectors are phased with two units of momentum (6). Principal correlator fits (11) are shown with colored bands, while excluded data are in gray. Data are shown for signal-to-noise ratios $\geq 2$. correlators for the higher excited states could not be resolved in such highly boosted frames, the resolution of the ground-state nucleon to at least $a p_{z}=6(2 \pi / L)$ marks a considerable improvement in the distillation/GEVP infrastructure for the study of hadron structure.

The results for our variational analyses of the unphased and phased bases for different momenta are summarized in Fig. 5, where we plot the extracted nucleon energies, together with expectations from both the continuum dispersion relation and the lattice dispersion relation for a free scalar particle. It is evident, even with the use of an extended operator basis and the correspondingly improved isolation of the ground state, distillation without phasing is unable to cleanly resolve the ground-state nucleon energy for $a p_{z}=4(2 \pi / L)$, where the signal is dominated by noise whether the single or variationally optimized operator is used.

Energies from the low-momentum phasing (5) were found to be consistent with those determined from the unphased GEVP, but are of substantially higher statistical quality. Most encouraging is that we are now able to map the ground-state nucleon dispersion relation up to $p_{z} \simeq$ $3 \mathrm{GeV}$ using the $\vec{\zeta}=2 \cdot \frac{2 \pi}{L} \hat{z}$ phased distillation space, even within the limited statistics. Moreover, significant uncertainty in the nucleon energies accrues only for the highest momenta $a p_{z}=\{7,8\} \times(2 \pi / L)$, where for discretization effects are considerable.

Confidence in our extracted nucleon energies is bolstered by a separate variational analysis of an extended operator basis containing only the spatially local interpolators, in particular the $N^{2} S_{S} \frac{1}{2}+$ and seven explicitly relativistic interpolators. These results are shown in red in Fig. 5 and are again consistent with the (un)phased determinations when using the $\mathcal{B}_{\vec{p} \neq 0}$ operator basis. The slightly higher values for the nucleon energies at large momenta are not surprising, as the purely local operator basis did not include negative-parity operators nor those of continuum spin $J>\frac{3}{2}$, certainly contaminating the true ground-state nucleon signal. Nonetheless, a consistent determination of the nucleon dispersion relation when using two distinct operator bases validates the union of distillation with momentum smearing, and in particular confirms that the addition of phase factors does not spoil the group theory required to construct our interpolating operators.

\section{MATRIX ELEMENTS AT HIGH MOMENTUM}

Hadron structure calculations within lattice QCD proceed through the calculation of matrix elements between hadrons of interest, implemented through the calculation of three-point, or higher, correlation functions. As emphasized in the Introduction, many of the key measures of hadron structure, such as the parton distribution functions computed in the LaMET, pseudo-PDF, or lattice-cross-section 


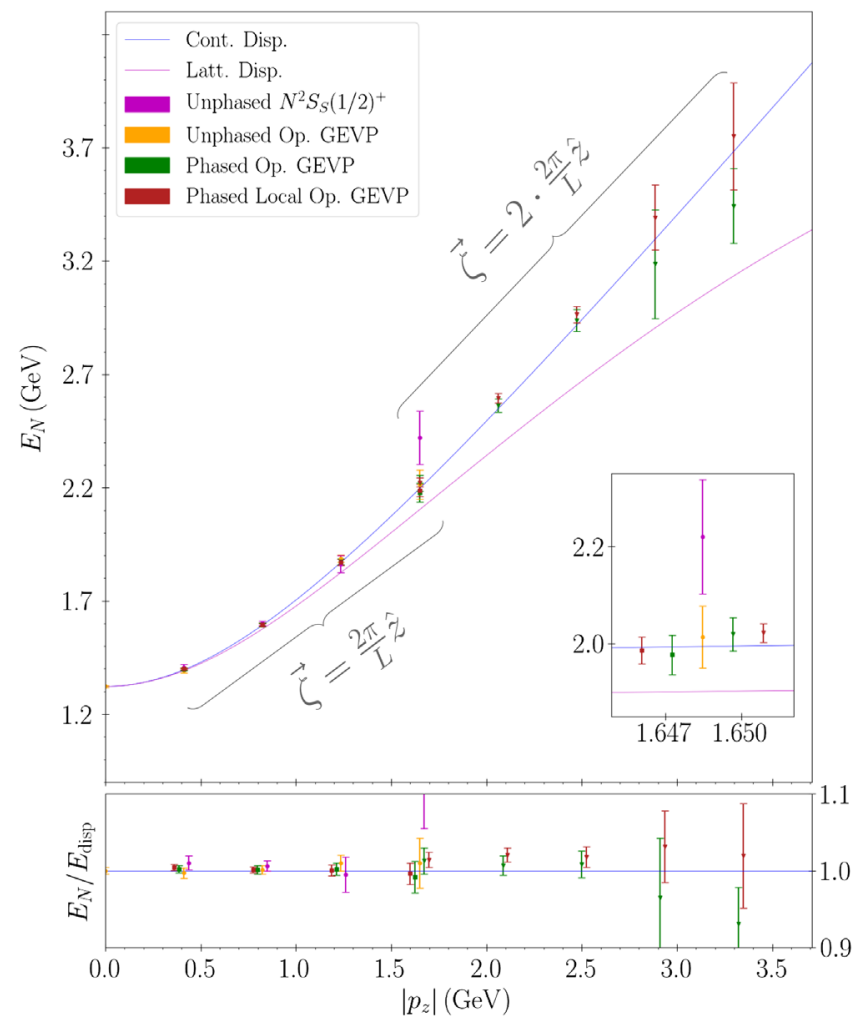

FIG. 5. The ground-state nucleon dispersion relation for the $a 094 m 358$ ensemble, together with expectations from the continuum dispersion relation (blue line) and free lattice scalar dispersion relation (purple line). Energies without the use of phasing are shown in magenta for a single, $N^{2} S_{S_{2}}{ }^{+}$operator and orange for the variational analysis using the bases $\mathcal{B}_{\vec{p}=0}, \mathcal{B}_{\vec{p} \neq 0}$. The energies obtained by applying the variational method on the phased $\mathcal{B}_{\vec{p}=\overrightarrow{0}}, \mathcal{B}_{\vec{p} \neq 0}$ bases are shown in green and for a basis of purely local operators in red. The squares and triangles denote the $\vec{\zeta}=\frac{2 \pi}{L} \hat{z}$ and $\vec{\zeta}=2 \cdot \frac{2 \pi}{L} \hat{z}$ phasing, respectively. The ground-state nucleon energies for momentum $a p_{z}=4(2 \pi / L)$ are shown in the inset plot, shifted for legibility. The lower panel emphasizes the differences between each method to obtain the ground-state nucleon energies, by normalizing each with respect to the continuum dispersion relation.

frameworks, require that the resulting three-point functions be computed for hadrons at as large a momentum, or over as large a range of momentum, as possible in order to have the best control over systematic uncertainties in their approaches. Thus the remainder of this paper is devoted to addressing this issue through the calculation of the nucleon isovector charges, in the forward direction, both for the nucleon at rest and for the nucleon in a moving frame of increasing boosts.

For our study of the nucleon charges, we use an ensemble at a somewhat lighter pion mass, which we denote by $a 094 m 278$, for which the relevant isovector current renormalization constants have been computed [40]; details of the ensemble are contained in Table I. At the lower values of momentum $\left[a p_{z}=\{0,1\} \times(2 \pi / L)\right]$, we use the vanilla form of distillation, without phasing. As we demonstrate below, at high momentum, where phasing is essential, we use two units of phasing, as implemented in Eq. (6). For $a p_{z}=4(2 \pi / L)$, we compare our results both with and without phasing as a consistency check of the method.

\section{A. Nucleon effective energies}

We begin by presenting in Fig. 6 the nucleon effective energies computed on the $a 094 m 278$ ensemble using ground-state interpolating operators obtained from the variational method with the $\mathcal{B}_{\vec{p}=\overrightarrow{0}}$ and $\mathcal{B}_{\vec{p} \neq 0}$ bases, following the procedure described for the $a 094 m 358$ ensemble. At all values of the momenta shown [i.e., $a p_{z} \leq 4(2 \pi / L)$ ] we show the results without phasing; for $a p_{z}=4(2 \pi / L)$, we also show the results using the phased eigenvectors, as described above. The need for phasing at this value of the momenta (green) and above is striking, where the plateau in the effective energy is clear at far greater temporal separations, and the resulting energy is far more precisely determined. We observe that at such a lighter pion mass, the variational method without phasing is insufficient to extract the ground-state nucleon energy for $a p_{z} \geq$ $4(2 \pi / L)$ (red), but arguably $a p_{z} \geq 3(2 \pi / L)$ (brown). We do not expound further on nucleon energies for this

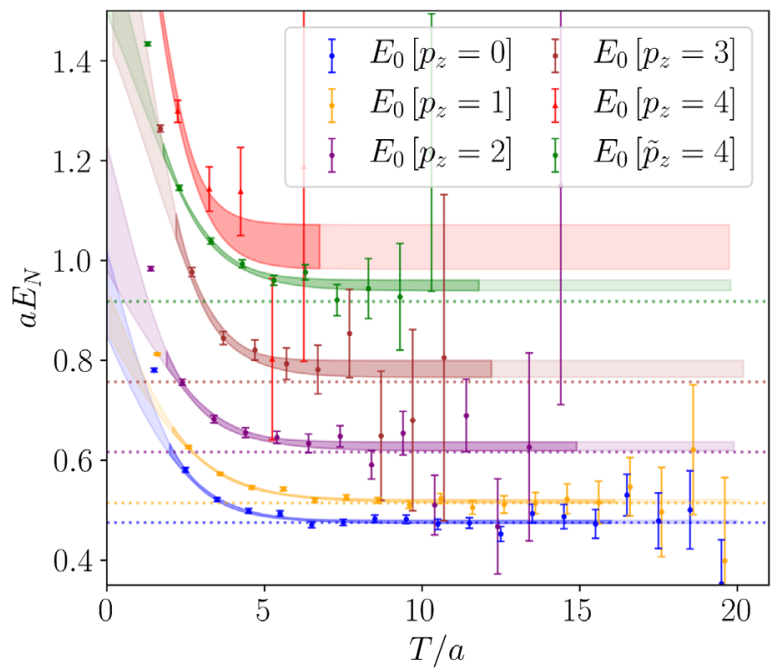

FIG. 6. Nucleon effective energies for the $a 094 m 278$ ensemble using a projected interpolator obtained from the $\mathcal{B}_{\vec{p}=\overrightarrow{0}}$ and $\mathcal{B}_{\vec{p} \neq \overrightarrow{0}}$ bases subduced into the relevant little group, together with continuum expectations (dashed), and two-state fits (bands), where in each case the darker region denotes the time series included in the fit. No phasing was used to extract the groundstate nucleon energy for lattice momenta $a p_{z} \in \mathbb{Z}_{5}$, while $a p_{z}=$ $4(2 \pi / L)$ was also determined with two units of phasing (6). In the case of $a p_{z}=4(2 \pi / L)$, the results with and without phased eigenvectors are shown as the green and red points, respectively, clearly demonstrating the need for phasing. Data shifted for legibility, and shown for signal-to-noise ratios greater than 1.35. 
ensemble; however, this demonstration underscores the need for variational improvement of a phased distillation space in order to study physical observables at high momenta.

\section{B. Charges}

We isolate forward isovector matrix elements by constructing nucleon three-point functions

$$
\begin{aligned}
C_{\text {3pt }}(T, \tau, \vec{p})= & \sum_{\vec{x}, \vec{y}, \vec{z}} e^{i \vec{p} \cdot(\vec{y}-\vec{x})} \mathcal{P}_{\beta \alpha}^{3 \mathrm{pt}} \\
& \times\left\langle\mathcal{N}_{\alpha}(\vec{y}, T) \mathcal{O}_{\Gamma}^{u-d}(\vec{z}, \tau) \overline{\mathcal{N}}_{\beta}(\vec{x}, 0)\right\rangle,
\end{aligned}
$$

with $\mathcal{O}_{\Gamma}^{u-d}$ an isovector insertion introduced at time $\tau$ between nucleon interpolators with temporal separation $T$, and $\mathcal{P}_{\beta \alpha}^{3 \mathrm{pt}}=\mathcal{P}^{2 \mathrm{pt}}\left(1+i \gamma_{5} \gamma_{3}\right)$ a $z$-polarized positive-parity projector. To study the asymptotic $0 \ll \tau \ll T$ behavior, we parametrize our two-point and three-point (13) correlation functions according to two-state fitting functionals

$$
\begin{gathered}
C_{\mathrm{fit}}^{2 \mathrm{pt}}(T)=e^{-E_{0} T}\left(a+b e^{-\Delta E T}\right), \\
C_{\mathrm{fit}}^{3 \mathrm{pt}}(T, \tau)=e^{-E_{0} T}\left(\mathcal{A}+\mathcal{B} e^{-\Delta E T}\right. \\
\left.+\mathcal{C} e^{-\Delta E^{\frac{T}{2}}} \cosh \left[\Delta E\left(\tau-\frac{T}{2}\right)\right]\right),
\end{gathered}
$$

where $\Delta E$ is the energy gap between the ground state $\left(E_{0}\right)$ and an effective first-excited $\left(E_{1}\right)$ state; $\mathcal{B}$ and $\mathcal{C}$, respectively, contain excited and transition matrix elements; and $\mathcal{A}$ contains the desired forward matrix element. Priors are again introduced to enforce the positivity of $\{a, b\}$. With these parametrizations, the desired groundstate matrix element is then $g_{00}^{\Gamma}=\mathcal{A} / a$ in the large- $T$ limit, as shown in [38]. We perform simultaneous correlated fits to the computed two-point and three-point correlators according to (14) and (15) to extract these parameters. Contact terms arising from the fermion action are excluded from the simultaneous fits by fitting in the windows $\tau_{\mathrm{fit}} / a \in[2, T-2]$ and $T_{\mathrm{fit}} / a \in\left[2, T_{\mathrm{fit}}^{\mathrm{max}}\right]$, where $T_{\mathrm{fit}}^{\max }$ is set by the maximal temporal range for which the associated principal correlators have signal-to-noise ratios exceeding unity:

(i) $(2 \pi / L)^{-1} a p_{z}=0: T_{\mathrm{fit}}^{\max }=16$

(ii) $(2 \pi / L)^{-1} a p_{z}=1: T_{\mathrm{fit}}^{\max }=16$

(iii) $(2 \pi / L)^{-1} a p_{z}=4$ [no phase]: $T_{\text {fit }}^{\max }=7$

(iv) $(2 \pi / L)^{-1} a p_{z}=4$ [phased]: $T_{\mathrm{fit}}^{\max }=12$.

When computing hadronic charges, the degree of excited-state contamination present in the three-point correlators for a given interpolator separation $T$ is often quantified (cf. $[38,40]$ ) via definition of an effective charge

$$
g_{\mathrm{eff}}^{\Gamma}(T, \tau)=C_{\Gamma}^{3 \mathrm{pt}}(T, \tau) / C_{\mathrm{fit}}^{2 \mathrm{pt}}(T),
$$

where the numerator is a three-point correlation function with inserted Dirac structure $\Gamma$ computed for intermediate times $\tau / a=[0, T-1]$ and $C_{\mathrm{fit}}^{2 \mathrm{pt}}(T)$ is the two-point function fit evaluated at the source-sink interpolator separation $T$. This ratio has the advantage of plateauing to $g_{00}^{\Gamma}$ as $\tau$ and $T-\tau$ become large, but is only useful in so far as $C_{\mathrm{fit}}^{2 \mathrm{pt}}$ is well determined and sufficiently captures the ground state. We find this ratio, particularly in the high-momentum frames considered, to be misleading when juxtaposed with the ratio of the simultaneous $C_{\Gamma}^{3 \mathrm{pt}}(T, \tau)$ and $C_{\mathrm{fit}}^{2 \mathrm{pt}}(T)$ fit. We instead illustrate the quality of our data by forming a direct ratio of the computed correlation functions

$$
R_{\Gamma}(T, \tau)=C_{\Gamma}^{3 \mathrm{pt}}(T, \tau) / C^{2 \mathrm{pt}}(T)
$$

All following figures depict these ratios (16) together with ratios of the fitted three-point and two-point functions for each $T / a$, as well as the extracted renormalized isovector charge indicated with a black line and gray error band. Data excluded from fits are in gray. All errors are determined via a simultaneous jackknife resampling of the data.

\section{CHARGE BEHAVIOR}

$$
\text { A. } g_{S}^{u-d}
$$

The isovector scalar $S=\bar{q} \frac{\tau^{3}}{2} q$ current within nucleon states decomposes trivially as

$$
\langle N|S| N\rangle=\frac{1}{2 M_{N}} \bar{u}_{N}\left(p_{f}\right) G_{S}^{u-d}\left(q^{2}\right) u_{N}\left(p_{i}\right),
$$

where $G_{S}^{u-d}$ is the isovector scalar form factor. The amplitude $G_{S}^{u-d}$ is Lorentz invariant and should thus be independent of the nucleon boost, absent excited-state, discretization, and finite-volume effects. In particular, in the forward limit one should, in principle, be able to access $G_{S}^{u-d}(0)=g_{S}^{u-d}$ regardless of frame. Figure 7 illustrates the $R_{S}(T, \tau)$ ratios needed to access the scalar charge and associated fits within our considered nucleon frames, demonstrating the degree to which this supposition is realized. In the rest frame a clear plateau is observed in the ratio by $T / a=10$, while determinations at larger values of $T / a$ deviate from this trend and exhibit increased uncertainty, the latter being consistent with the observed variability of the nucleon effective energies at these same times. Most notable is a reduction in value and uncertainty of $g_{S}^{u-d}$ when compared with standard, high statistics, smearing schemes on the same $a 094 m 278$ ensemble. Namely in [40], it was found $g_{S}^{u-d}=0.990(89)$ — the use of distillation has led to a more precise determination by $\sim 75 \%$.

Considering the $a p_{z}=(2 \pi / L)$ frame, we observe statistical consistency with the $a p_{z}=0$ determination, with a plateau emerging for $T / a \sim 10-12$. The expected increase 


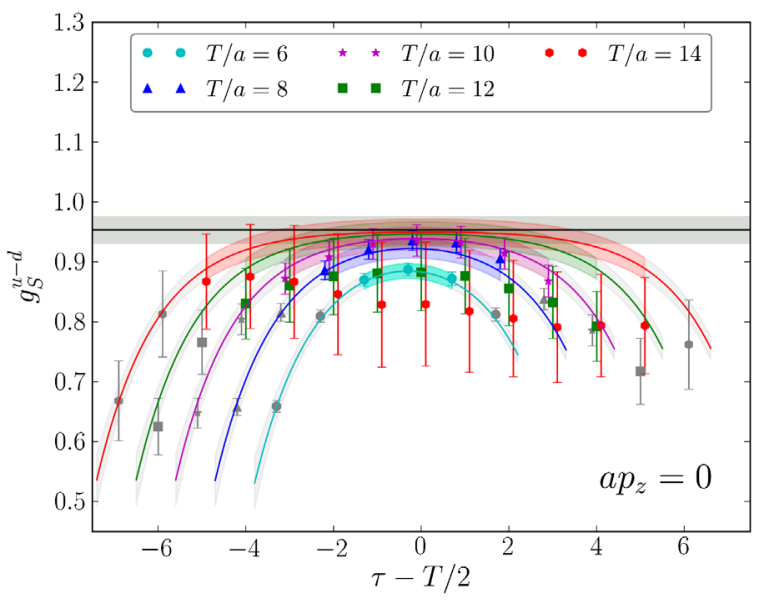

(a)

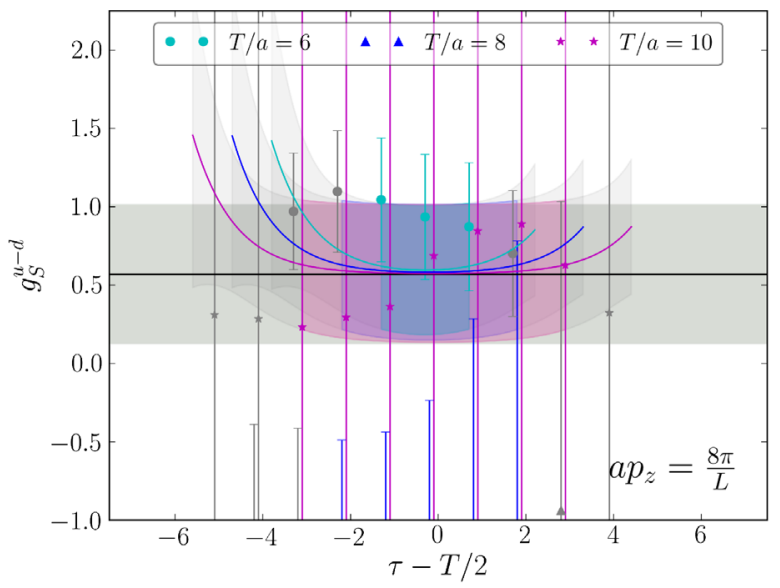

(c)

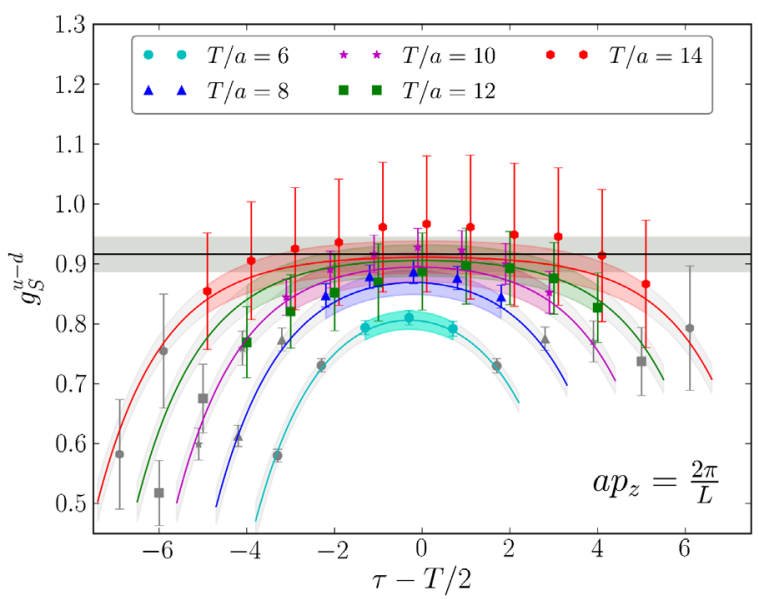

(b)

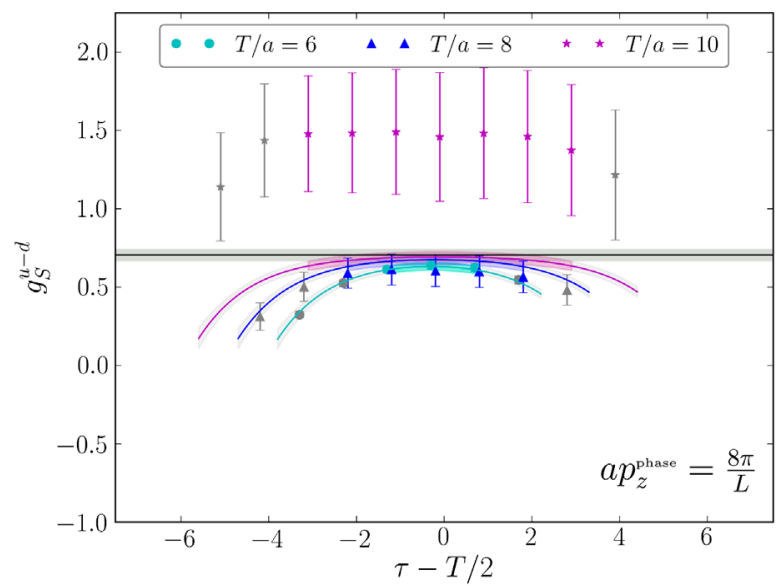

(d)

FIG. 7. Extracted renormalized $R_{S}(T, \tau)$ and isovector scalar charges for momenta (a) $a p_{z}=0$, (b) $a p_{z}=(2 \pi / L)$, (c) $a p_{z}=4 \times(2 \pi / L)$ without phasing, and (d) $a p_{z}=4 \times(2 \pi / L)$ with two units of allowed lattice momentum applied to the eigenvectors. Variationally improved operators were used within each momentum channel.

of excited-state contamination is evident in Fig. 7(b), where there exists greater curvature of the ratio data for a given $T$ and the difference between each $R_{S}(T, \tau)$ plateau and the asymptotic charge is seen to increase relative to the rest case. This amounts to marked increases in $\mathcal{B}$ and $\mathcal{C}$ of Eq. (15) which capture excited-state $\left\langle N^{\prime}|S| N^{\prime}\right\rangle$ and transition $\left\langle N^{\prime}|S| N\right\rangle$ matrix elements, respectively.

Without the introduction of appropriate momentum phases into the distillation space, attempts to access the scalar charge in a highly boosted frame are utterly meaningless [Fig. 7(c)]. Isolation of the scalar charge in the $a p_{z}=4 \times(2 \pi / L)$ frame is, however, dramatically improved when a phased distillation space is used. The statistical precision of the $R_{S}(T, \tau)$ data improves considerably, provided the two-point function is well determined. However, the extracted charge is dubious - the phased determination differs by $25 \%$ from the average of the $a p_{z}=\{0,1\} \times(2 \pi / L)$ cases. The close proximity of the $R_{S}(T, \tau)$ plateaus for each $T / a$ and the asymptotic charge suggest that at the level of the two-state fits considered herein, the first excited-state matrix element is small. However, without performing $R_{S}(T, \tau)$ computations for additional $T / a$ and performing higher state fits, this cannot be rigorously confirmed. We do point out the statistical noise evident in the $T / a=10$ data is not surprising, as the phased two-point function loses its signal at $T / a \sim 10$ (cf. Fig. 6). Furthermore, determinations of $Z_{S}$ found in [40] vary below the $2 \%$ level and thus also cannot explain the observed discrepancy. One may be tempted to attribute this dramatic difference to a mixing of the scalar current with the derivative of the vector current $D_{\mu}\left\{\bar{\psi} \gamma_{\mu} \psi(x) e^{-i q \cdot x}\right\}$. Given the explicit zero threemomentum transfer with the probing current, it is evident this derivative mixing is possible only when $q_{4} \neq 0$ or when unwanted excited-to-ground state transitions are present. This possibility is captured by $\mathcal{C}$ of (15) and is reflected in the overall curvature of $R_{S}(T, \tau)$ rather than vertical shifts of the computed matrix element. We are left 
TABLE II. Renormalized isovector scalar charge determined at rest and in boosted frames.

\begin{tabular}{lllcl}
\hline$g_{\Gamma}$ & $a p_{z}=0$ & $a p_{z}=2 \pi / L$ & $a p_{z}=8 \pi / L$ & $a p_{z}^{\text {phase }}=8 \pi / L$ \\
\hline$g_{S}^{u-d}$ & $0.953(22)$ & $0.916(28)$ & $0.57(44)$ & $0.705(35)$ \\
$\chi_{r}^{2}$ & 0.920 & 1.010 & 12.482 & 2.037 \\
\hline \hline
\end{tabular}

to attribute this puzzling discrepancy to statistical fluctuations and the lack of additional $T / a$ data. As will be shown, the other charges we explore exhibit much greater consistency in the studied momentum frames, and observed deviations can be attributed to known systematic effects. Table II catalogs the isolated scalar charges and the correlated figure of merit for the simultaneous fits of each frame.

\section{B. $g_{V}^{u-d}$}

Among the currents considered, the vector current $V_{\mu}=$ $\bar{q} \gamma_{\mu} \frac{\tau^{3}}{2} q$ is unique given that it is a conserved quantity in the continuum. Our decision to adopt purely local currents in this work necessarily violates this conservation. However, the derived vector current renormalization constant [40] reestablishes the desired conservation up to quadratic corrections in the lattice spacing-namely, $Z_{V} g_{V, \text { bare }}^{u-d}=$ $1+\mathcal{O}\left(a^{2}\right)$. Considering the vector current Lorentz structure between the ground-state nucleon and an arbitrary state $N^{\prime}$ with nucleon quantum numbers

$$
\begin{aligned}
\left\langle N^{\prime}\left|V_{\mu}\right| N\right\rangle= & \bar{u}_{N^{\prime}}\left(p_{f}\right)\left[F_{1}^{u-d}\left(q^{2}\right)\left(\gamma_{\mu}-\frac{q_{\mu}}{q^{2}} \not \phi\right)\right. \\
& \left.+\frac{\sigma_{\mu \nu} q_{\nu}}{M_{N^{\prime}}+M_{N}} F_{2}^{u-d}\left(q^{2}\right)\right] u_{N}\left(p_{i}\right),
\end{aligned}
$$

it is clear for $\vec{q}=0$ the temporal component of the vector current simply yields the baryon number of the nucleon and all its excitations. A useful sanity check then for the phasing considered herein is to ensure the renormalized $g_{V}^{u-d}$ is unity in the $V_{4}=\bar{q} \gamma_{4} q$ channel for each forward frame considered. As illustrated in Figs. 8, 9(b), and 9(f), we indeed find $Z_{V} g_{V_{4} \text {,bare }}^{u-d}$ to be unity and temporally invariant, most notably even as the nucleon momentum is increased and phasing is employed. A highly boosted nucleon interpolator without phasing exhibits poor overlap with the ground-state nucleon [Fig. 9(d)] and is sufficiently noisy such that $Z_{V} g_{V_{4} \text {,bare }}^{u-d} \neq 1$. The extracted $g_{V_{4}}^{u-d}$ are presented in Table III, with consistent determinations observed in the $a p_{z}=\{0,1\} \times(2 \pi / L)$ and $a p_{z}^{\text {phase }}=$ $4(2 \pi / L)$ momentum channels.

Nonzero nucleon momenta while still with $\vec{q}=0$ opens the $V_{3}=\bar{q} \gamma_{3} \frac{\tau^{3}}{2} q$ channel as an additional means to quantify the ground-state Dirac form factor $F_{1}^{u-d}(0)$. However, any such attempt to isolate the ground-state Dirac form factor $F_{1}^{u-d}$ signal will be contaminated with

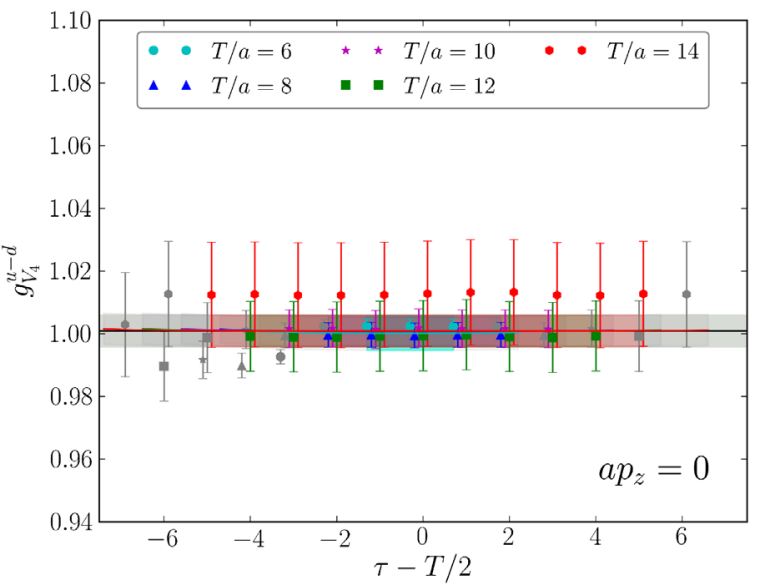

FIG. 8. Extracted renormalized $R_{V_{4}}(T, \tau)$ and isovector vector charges determined for momenta $a p_{z}=0$. A variationally improved operator was used in these determinations.

the transition form factor $F_{2}^{u-d}\left(q^{2}\right)$ signal in proportion to $q_{4} /\left(M_{N^{\prime}}+M_{N}\right)$. In the ideal scenario that excited states are completely removed, the energy transfer $q_{4}$ will vanish and $F_{1}^{u-d}(0)$ can be directly accessed with $V_{3}$. Figure 9(a) illustrates $R_{V_{3}}(T, \tau)$, which features a clear dependence on $\{T, \tau\}$ and whose asymptotic limit differs from $g_{V_{4}}^{u-d}$ by $\sim 8 \%$, together indicating the presence of excited states. Thus absent a dedicated study and subsequent removal of the $F_{2}^{u-d}$ contamination, the best we can extract here is $F_{1}^{u-d}\left(q^{2}\right)-\frac{q_{4} \gamma_{4}}{M_{N^{\prime}}+M_{N}} F_{2}^{u-d}\left(q^{2}\right)$-which we will denote as $g_{V_{3}}^{u-d}$ for brevity. To the extent this pollution is unchanging in other forward frames is borne out in Figs. 9(c) and 9(e). As for the scalar charge, the unphased $a p_{z}=4(2 \pi / L)$ determination is meaningless and is dominated by uncertainty in the unaltered two-point function. The phased $a p_{z}=4(2 \pi / L)$ determination, although statistically consistent with $g_{V_{4}}^{u-d}$, is constrained by only two values of $T$ and is characterized by a curious flip in concavity of $R_{V_{3}}(T, \tau)$. As this dependence is captured by $\mathcal{C}$ of Eq. (15), it is clear the effect of phasing has apparently identified the conjugate of the ground-to-first-excited state transition. This behavior warrants repeated calculations for additional values of $T / a$ with increased statistics to elucidate whether this behavior is merely fluctuations or a clear trend. That said, the $R_{V_{3}}(T, \tau)$ appears to be trending below unity within the well-determined values of $T / a$. Results of these simultaneous fits are cataloged in Table IV.

$$
\text { C. } g_{A}^{u-d}
$$

The axial charge of the nucleon is perhaps the most enigmatic of the isovector charges given its long history as a benchmark in LQCD, and only recent efforts falling to within $1 \%$ of experiment [44-46]. At zero momentum the nucleon expectation of the axial current is vanishing except for components along the direction of polarization. 


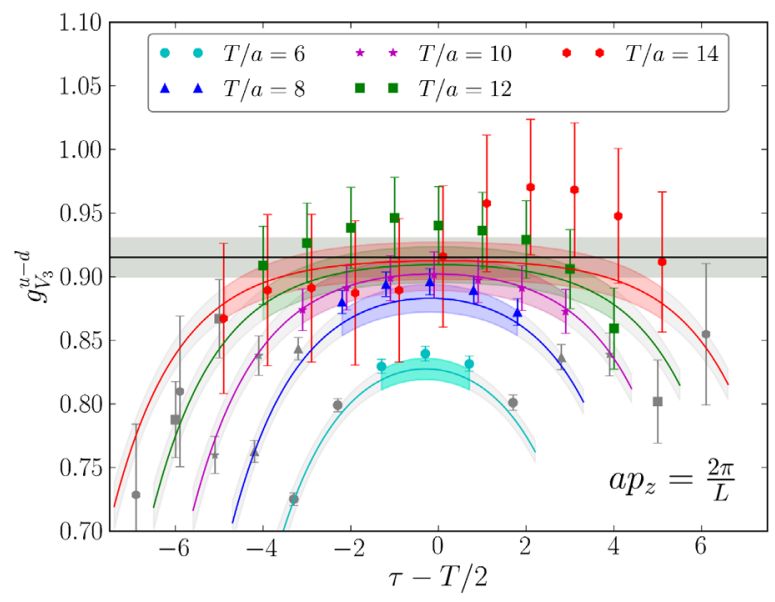

(a)

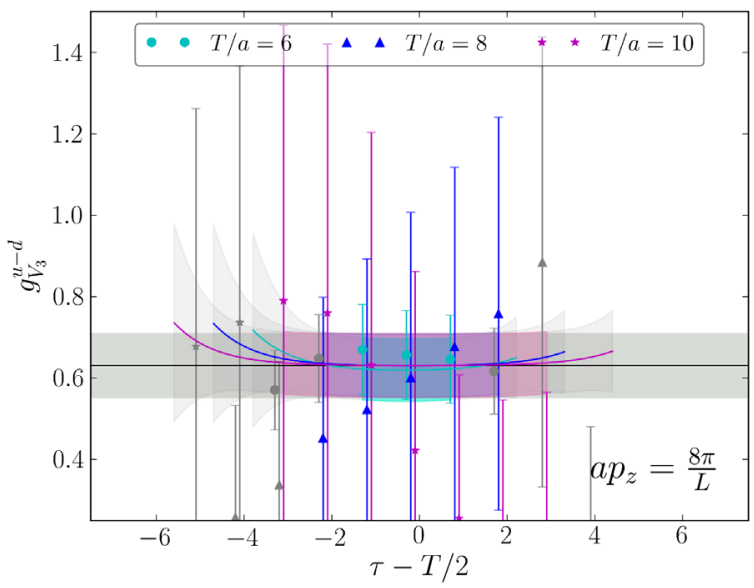

(c)

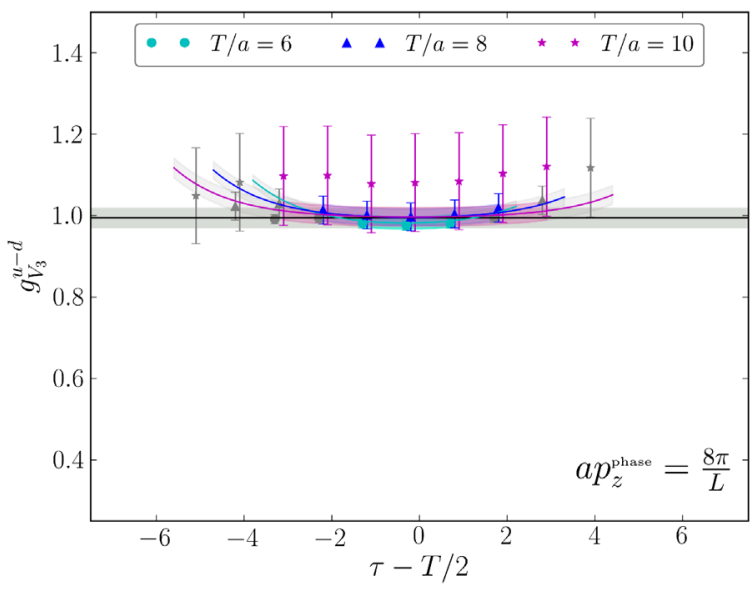

(e)

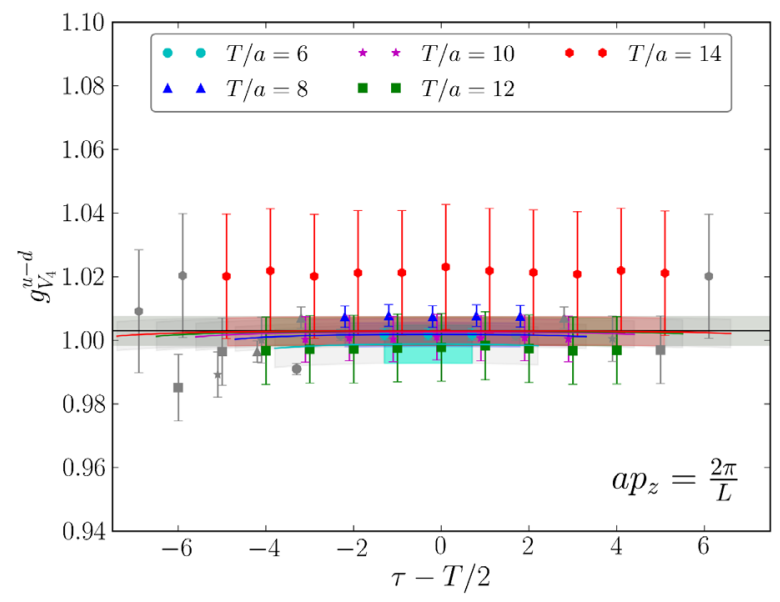

(b)

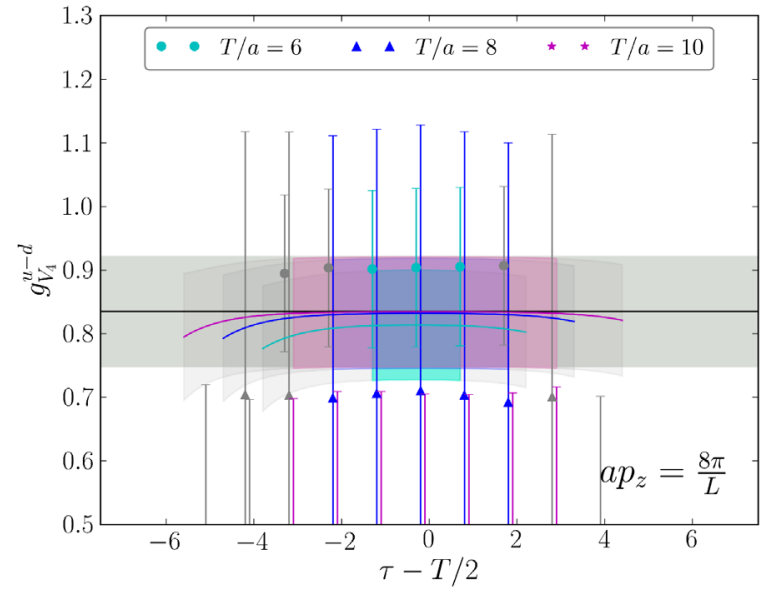

(d)

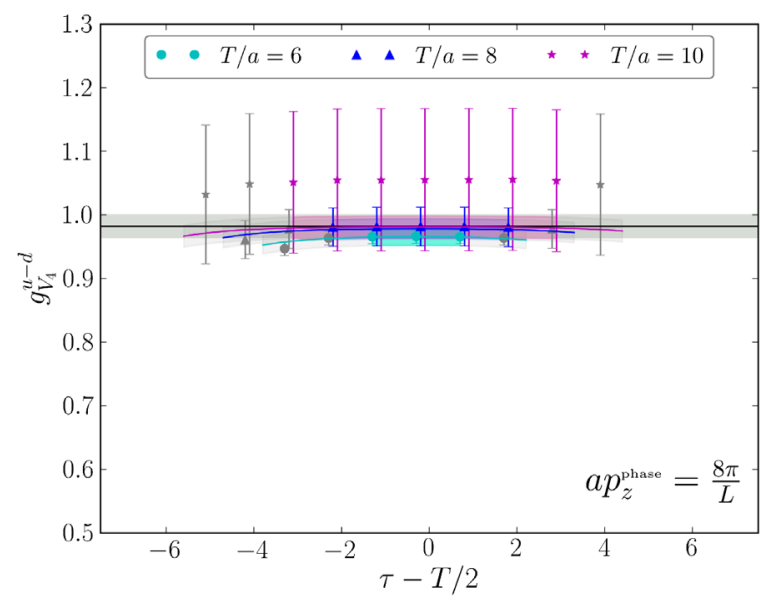

(f)

FIG. 9. Extracted renormalized $R_{V_{u}}(T, \tau)$ and isovector vector charges determined from $\gamma_{3}$ (left panel) and $\gamma_{4}$ (right panel) insertions. External nucleon momentum according to (a),(b) $a p_{z}=(2 \pi / L)$; (c),(d) $a p_{z}=4(2 \pi / L)$ without phasing; and (e),(f) $a p_{z}=4(2 \pi / L)$ with two units of allowed lattice momenta applied to the eigenvectors. Variationally improved operators were used within each momentum channel. 
TABLE III. Renormalized isovector vector charges determined via $\gamma_{4}$ at rest and in boosted frames.

\begin{tabular}{llccc}
\hline \hline$g_{\Gamma}$ & $a p_{z}=0$ & $a p_{z}=2 \pi / L$ & $a p_{z}=8 \pi / L$ & $a p_{z}^{\text {phase }}=8 \pi / L$ \\
\hline$g_{V_{4}}^{u-d}$ & $1.001(5)$ & $1.003(4)$ & $0.84(9)$ & $0.982(18)$ \\
$\chi_{r}^{2}$ & 0.901 & 1.767 & 12.317 & 1.902 \\
\hline \hline
\end{tabular}

TABLE IV. Renormalized $g_{V_{3}}^{u-d}$ determined via $\gamma_{3}$ in boosted frames. By definition, $g_{V_{3}}^{u-d}=0$ at rest.

\begin{tabular}{lcccc}
\hline \hline$g_{\Gamma}$ & $a p_{z}=0$ & $a p_{z}=2 \pi / L$ & $a p_{z}=8 \pi / L$ & $a p_{z}^{\text {phase }}=8 \pi / L$ \\
\hline$g_{V_{3}}^{u-d}$ & $\ldots$ & $0.915(15)$ & $0.63(8)$ & $0.995(23)$ \\
$\chi_{r}^{2}$ & $\cdots$ & 1.216 & 12.544 & 2.150 \\
\hline \hline
\end{tabular}

Thus for our $z$-polarized nucleons, we must use $\gamma_{3} \gamma_{5}$ at rest to access $g_{A}^{u-d}$ — which we denote as $g_{A_{3}}^{u-d}$. Together with a Lorentz decomposition of the axial current

$$
\begin{aligned}
\left\langle N\left|A_{\mu}\right| N\right\rangle= & \bar{u}_{N}\left(p_{f}\right)\left[\gamma_{\mu} \gamma_{5} G_{A}^{u-d}\left(q^{2}\right)\right. \\
& \left.-i \frac{q_{\mu}}{2 M_{N}} \gamma_{5} \tilde{G}_{P}^{u-d}\left(q^{2}\right)\right] u_{N}\left(p_{i}\right)
\end{aligned}
$$

and $\vec{q}=0$, it is evident the axial matrix element at rest receives contributions only from the axial form factor and not the induced pseudoscalar form factor. We plot in Fig. 10 the renormalized $R_{\gamma_{3} \gamma_{5}}(T, \tau)$ and $g_{A_{3}}^{u-d}$ isolated at rest from our simultaneous fits. We observe noticeable contamination from excited states for $T / a=\{6,8\}$, but broad consistency for the remaining $T / a$ values. The observed $\sim 7 \%$ deviation from the experimental value of 1.2756(13) [47] is not the focus of this work, but is conventionally attributed to finitevolume effects and excited states. In fact, it has been observed [48] that the $\gamma_{3} \gamma_{5}$ channel is particularly sensitive

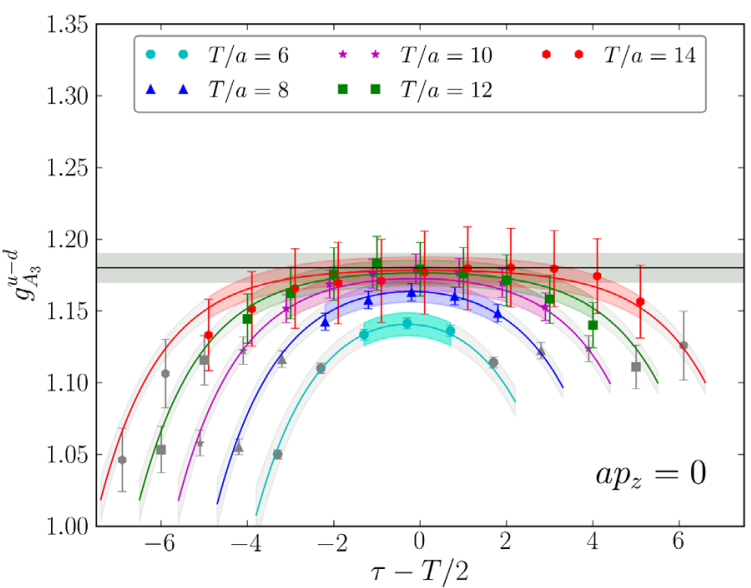

FIG. 10. Extracted renormalized $R_{\gamma_{3} \gamma_{5}}(T, \tau)$ and isovector axial charge determined via $\gamma_{3} \gamma_{5}$ at rest. A variationally optimized operator was used in these determinations. to closely spaced excited states, which when incorrectly identified leads to not only a discrepancy of $g_{A_{3}}^{u-d}$ with experiment but also a violation of the operator derived partially conserved axial current relation. Our deviation of $g_{A_{3}}^{u-d}$ from experiment is, however, consistent with [40], where with standard smearing schemes on the same $a 094 m 278$ ensemble it was found $g_{A_{3}}^{u-d}=1.208(33)$. We emphasize the use of distillation has led to a threefold reduction in uncertainty.

Other potential systematic errors in computations of $g_{A_{3}}^{u-d}$ have long been explored, such as use of $\mathcal{O}(a)$-improved currents [49]. In that work, however, it was found that use of an $\mathcal{O}(a)$-improved axial current only mildly improved the experiment-lattice discrepancy, bolstering the presumed preponderance of excited-state and finite-volume effects. These same authors explored the degree to which $g_{A_{3}}^{u-d}=g_{A_{4}}^{u-d}$ could be satisfied, just as we now explore based on Eq. (18).

Figure 11 illustrates the $R_{\gamma_{3} \gamma_{5}}(T, \tau)$ and $R_{\gamma_{4} \gamma_{5}}(T, \tau)$ ratios isolated in the boosted frames we have considered. As with the scalar and vector charges, the lack of phasing at highmomentum degrades the two-point correlator such that the resulting matrix element signals contain essentially no information [Figs. 11(c) and 11(d)]. Compared to the rest frame, we observe $\sim 3 \%$ difference in $g_{A_{3}}^{u-d}$ when computed in the $a p_{z}=(2 \pi / L)$ frame. This difference is indicative of $q^{2} \neq 0$, despite $\vec{q}=0$, and hence mild radiative transitions with excited states affect this determination. Furthermore, we do observe a dramatic difference of $\sim 15 \%$ between the determination of $g_{A_{3}}^{u-d}$ and $g_{A_{4}}^{u-d}$ in the $a p_{z}=(2 \pi / L)$ frame. This is unsurprising given the observation of $q^{2} \neq 0$ in the moving $\gamma_{3} \gamma_{5}$ channel, all but ensuring the outsized influence of $\tilde{G}_{P}^{u-d}[50,51]$. The increased separation between each $R_{\gamma_{4} \gamma_{5}}(T, \tau)$ in Fig. 11(b) again points to this increased excited-state contamination. We do remark that despite the different vertical scales chosen in Figs. 11(a) and 11(b), the fitted energy gap $\Delta E$ is consistent within error. The momentum smeared $a p_{z}=4(2 \pi / L)$ charges [Figs. 11(e) and 11(f)] again exhibit improved statistical quality, yet superficially appear to agree with each other and oddly with experiment. Each determination does not, however, seem to indicate a plateau in $R_{\Gamma}(T, \tau)$ has been found, especially in light of the noisy $R_{\Gamma}(T=10, \tau)$ determinations. Moreover, the $R_{\gamma_{3} \gamma_{5}}(T, \tau)$ and $R_{\gamma_{4} \gamma_{5}}(T, \tau)$ ratios are clearly trending away from each other within the illustrated data, and suggest the extracted charges in this phased frame would indeed be distinct were calculations performed with improved statistics and, especially, finer $T / a$. The results for our simultaneous fits for the $g_{A_{3}}^{u-d}$ and $g_{A_{4}}^{u-d}$ axial charges are presented in Tables V and VI, respectively.

$$
\text { D. } g_{T}^{u-d}
$$

The isovector tensor current within nucleon states induces the following form factor decomposition: 


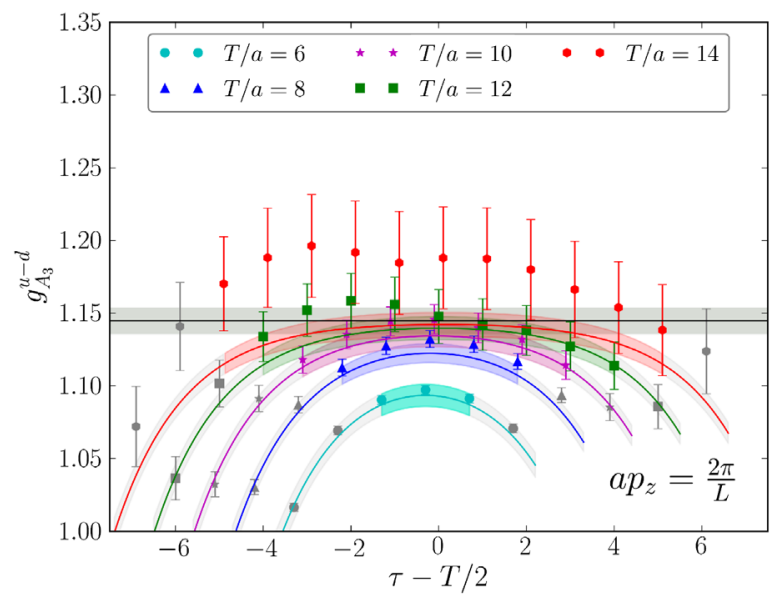

(a)

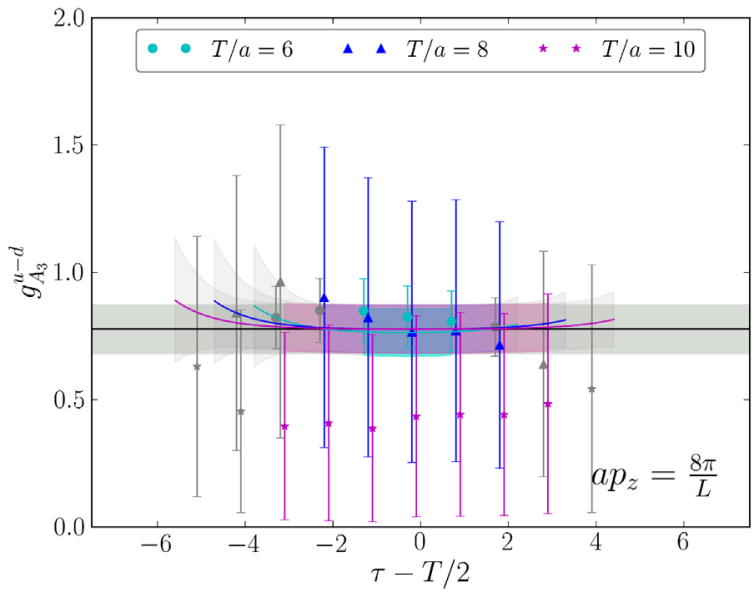

(c)

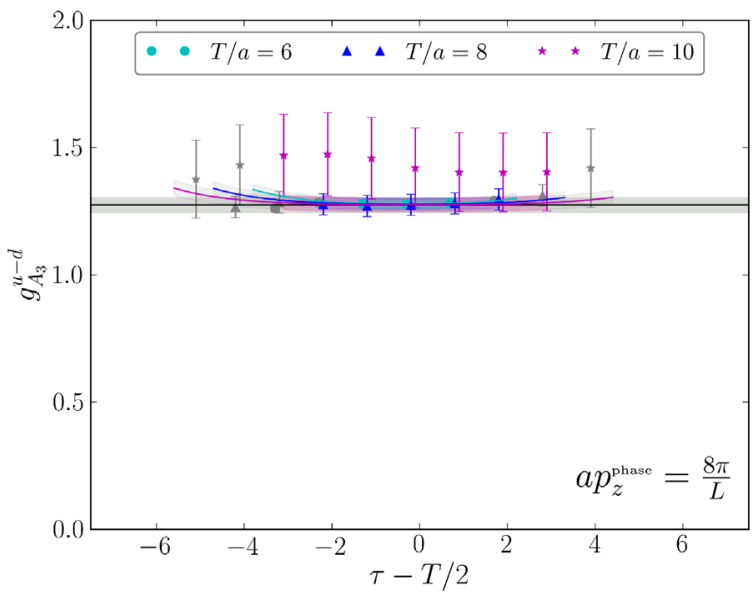

(e)

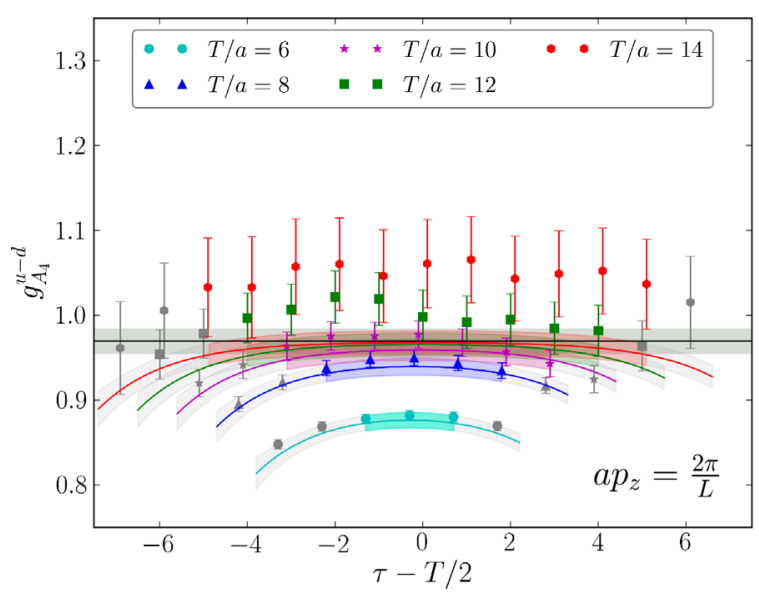

(b)

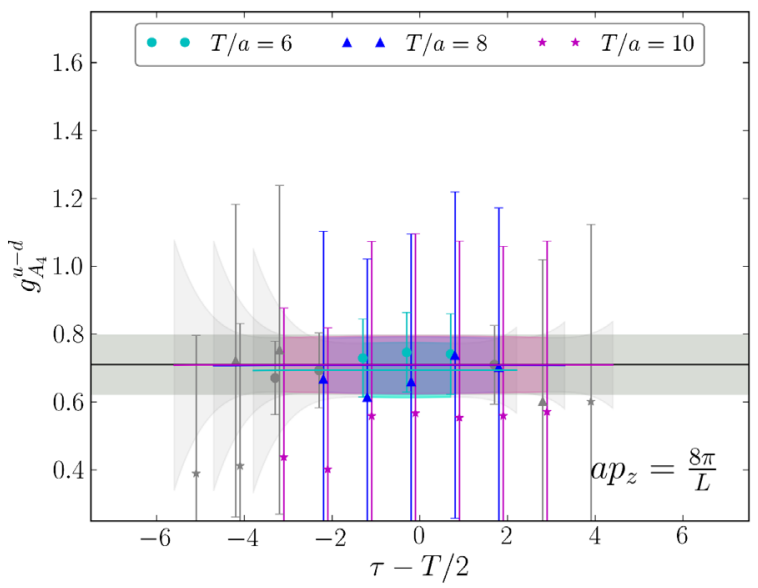

(d)

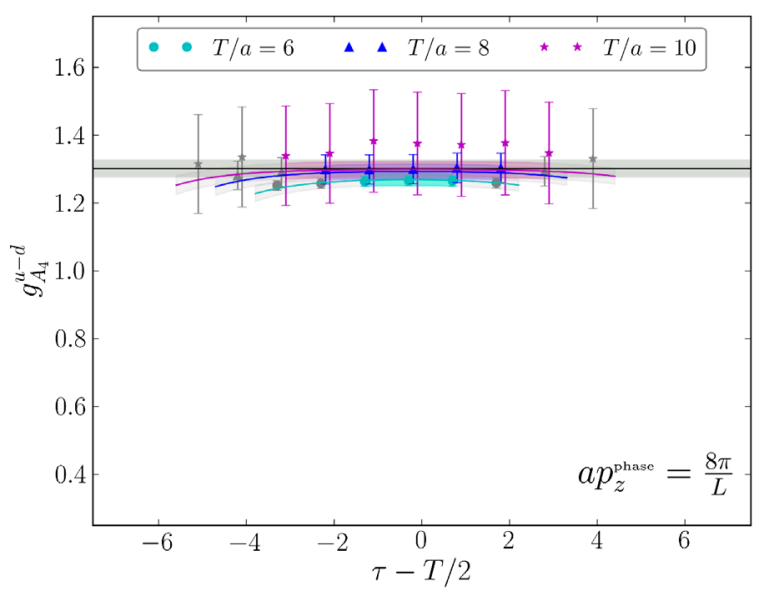

(f)

FIG. 11. Extracted renormalized $R_{A_{\mu}}(T, \tau)$ and isovector axial charges using $\gamma_{3} \gamma_{5}$ (left) and $\gamma_{4} \gamma_{5}$ (right). External nucleon momentum according to (a),(b) $a p_{z}=(2 \pi / L)$; (c), (d) $a p_{z}=4(2 \pi / L)$ without phasing; and (e),(f) $a p_{z}=4(2 \pi / L)$ with two units of allowed lattice momentum applied to the eigenvectors. Variationally improved operators were used within each momentum channel. 
TABLE V. Renormalized isovector axial charge determined via $\gamma_{3} \gamma_{5}$ at rest and in boosted frames.

\begin{tabular}{lllcl}
\hline \hline$g_{\Gamma}$ & $a p_{z}=0$ & $a p_{z}=2 \pi / L$ & $a p_{z}=8 \pi / L$ & $a p_{z}^{\text {phase }}=8 \pi / L$ \\
\hline$g_{A_{3}}^{u-d}$ & $1.18(1)$ & $1.145(9)$ & $0.8(1)$ & $1.275(29)$ \\
$\chi_{r}^{2}$ & 1.255 & 1.421 & 12.301 & 2.761 \\
\hline \hline
\end{tabular}

TABLE VI. Renormalized isovector axial charges determined via $\gamma_{4} \gamma_{5}$ in boosted frames. By definition, $g_{A_{4}}^{u-d}=0$ at rest.

\begin{tabular}{lcccc}
\hline \hline$g_{\Gamma}$ & $a p_{z}=0$ & $a p_{z}=2 \pi / L$ & $a p_{z}=8 \pi / L$ & $a p_{z}^{\text {phase }}=8 \pi / L$ \\
\hline$g_{A_{4}}^{u-d}$ & $\ldots$ & $0.970(14)$ & $0.71(9)$ & $1.302(24)$ \\
$\chi_{r}^{2}$ & $\cdots$ & 1.148 & 12.353 & 1.990 \\
\hline \hline
\end{tabular}

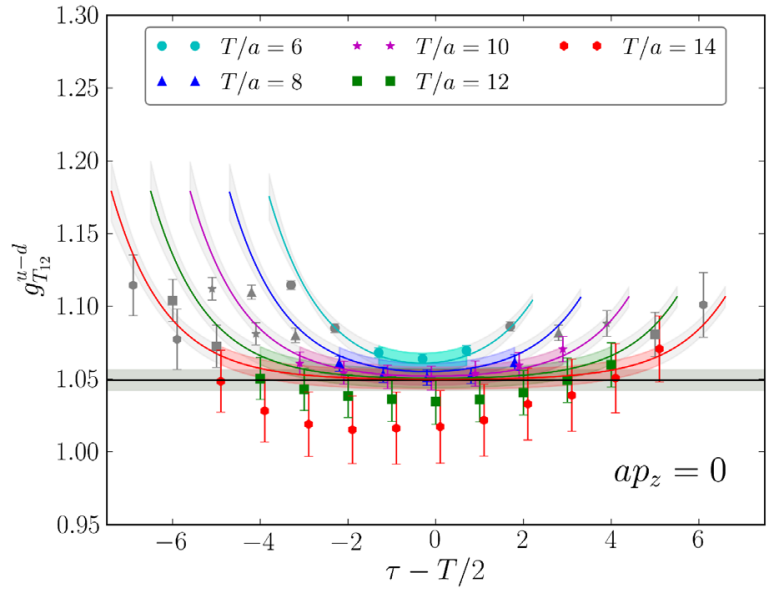

(a)

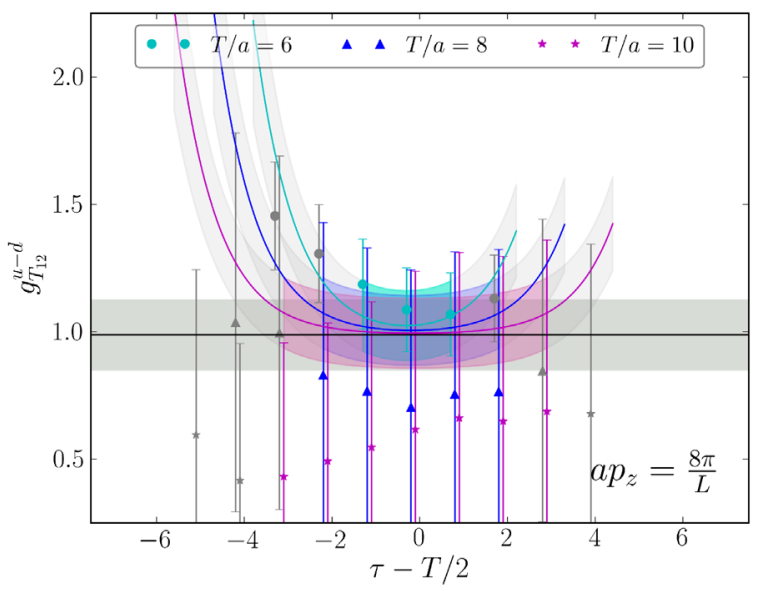

(c)

$$
\begin{aligned}
\left\langle N\left|T_{\mu \nu}\right| N\right\rangle= & \bar{u}_{N}\left(p_{f}\right)\left[i \sigma_{\mu \nu} A_{10}^{u-d}\left(q^{2}\right)+\frac{\left[\gamma_{\mu}, q_{\nu}\right]}{2 M_{N}} B_{10}^{u-d}\left(q^{2}\right)\right. \\
& \left.+\frac{\left[P_{\mu}, q_{\nu}\right]}{2 M_{N}^{2}} \tilde{A}_{10}^{u-d}\left(q^{2}\right)\right] u_{N}\left(p_{i}\right)
\end{aligned}
$$

where $T_{\mu \nu}=\bar{q} i \sigma_{\mu \nu} \frac{\tau^{3}}{2} q$ and $P=p_{f}+p_{i}$. At rest only the $T_{12}=\bar{q} \sigma_{12} \frac{\tau^{3}}{2} q$ matrix element is nonvanishing; apart from kinematic factors, this particular tensor current continues to be nonvanishing within the nucleon in motion. This has the fortunate consequence that all form factors outside the desired $A_{10}^{u-d}\left(q^{2}\right)$, where $g_{T_{12}}^{u-d} \equiv A_{10}^{u-d}(0)$, do not contribute to the matrix element signal. We indeed find $g_{T_{12}}^{u-d}$ determined in each momentum frame to be statistically consistent across the boosts considered, and in the case of $a p_{z}=\{0,1\} \times(2 \pi / L)$ the charge is especially well determined and in fantastic mutual agreement

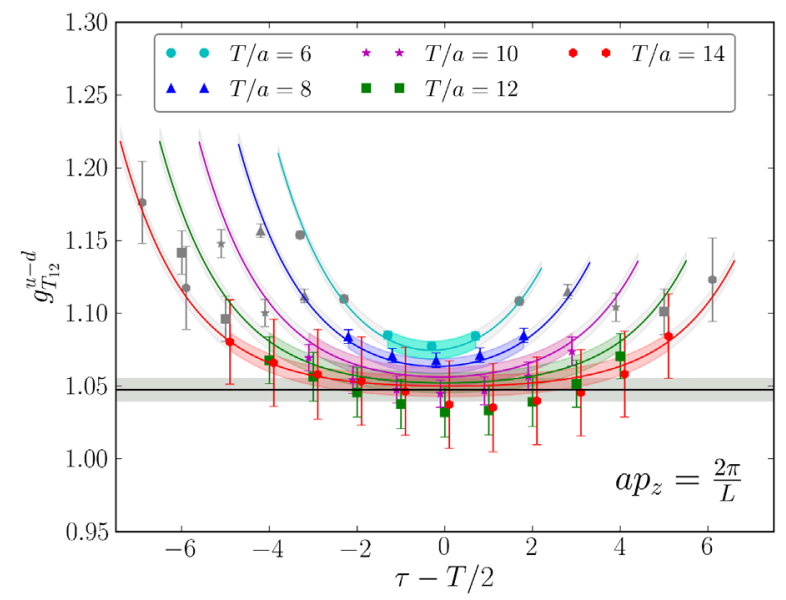

(b)

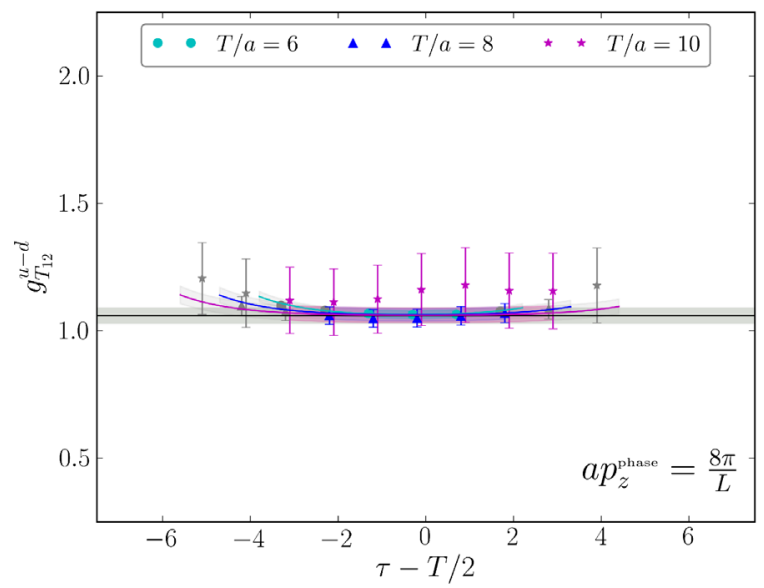

(d)

FIG. 12. Extracted renormalized $R_{T_{12}}(T, \tau)$ and isovector tensor charge determined for (a) $a p_{z}=0$, (b) $a p_{z}=(2 \pi / L)$, (c) $a p_{z}=4(2 \pi / L)$ without phasing, and (d) $a p_{z}=4(2 \pi / L)$ with two units of allowed lattice momentum applied to the eigenvectors. Variationally improved operators were used within each momentum channel. 
TABLE VII. Renormalized isovector tensor charges determined via $T_{12}$ at rest and in boosted frames.

\begin{tabular}{llccc}
\hline$g_{\Gamma}$ & $a p_{z}=0$ & $a p_{z}=2 \pi / L$ & $a p_{z}=8 \pi / L$ & $a p_{z}^{\text {phase }}=8 \pi / L$ \\
\hline$g_{T_{12}}^{u-d}$ & $1.049(7)$ & $1.048(8)$ & $0.99(14)$ & $1.06(3)$ \\
$\chi_{r}^{2}$ & 1.267 & 1.064 & 12.603 & 1.999 \\
\hline \hline
\end{tabular}

[see Figs. 12(a) and 12(b)]. The lack of a clean signal for $g_{T_{12}}^{u-d}$ for $a p_{z}=4(2 \pi / L)$ [Fig. 12(c)] is by now expected and underscores the need for phasing at high momentum [Fig. 12(d)]. We note the slightly larger, though no less consistent, value found for $g_{T_{12}}^{u-d}$ in the phased $a p_{z}=$ $4(2 \pi / L)$ frame appears to be a result of the noisy $T / a=10$ data. We anticipate future calculations with improved statistics will help to bring down this value. The ratios $R_{T_{12}}(T, \tau)$ and simultaneous fit results are compared in Fig. 12 and the extracted tensor charges are gathered in Table VII. In the interest of completeness, we note our best determined $g_{T_{12}}^{u-d}$ is $\sim 8 \%$ larger than $g_{T_{12}}^{u-d}=$ $0.973(36)$ determined in [40], yet several times more precise.

Summarizing, repeated calculations on lattice ensembles of varying lattice spacings and fixed physical volumes are necessary to rigorously pin down the size of discretization effects on these results. A dedicated study of contributing form factors is underway and will further facilitate the conclusions herein. Of course, a further source of discrepancy of all computed charges are finite-volume effects. One would expect finite-volume effects to be minor for these charges, given that the $a 094 m 278$ ensemble is characterized by $m_{\pi} L \simeq 4.24$. Nonetheless, calculations at different physical volumes required to confirm this expectation are planned.

\section{CONCLUSIONS}

We have expounded upon the seminal Gaussian momentum smearing scheme developed by Bali et al., demonstrating momentum space overlaps of distilled interpolators can likewise be improved by introducing appropriate spatial phase factors onto eigenvectors of the gaugecovariant Laplacian. We elected to introduce phases onto a precomputed eigenvector basis, rather than rotating the underlying gauge transporters. Consequently, the introduced phase factors were limited to allowed lattice momenta in the numerical investigations herein. Regardless of when the phase factors are introduced, all components forming the scaffolding of a distillationsmeared correlation function (e.g., elementals and perambulators) must be recomputed. This motivated our choice to smear precomputed eigenvectors.
We established the efficacy of this approach by isolating the ground-state nucleon dispersion relation using a standard eigenvector basis and two modified bases, modified with one and two units of allowed lattice momenta, respectively. Despite variational optimization of unmodified interpolators within the $J^{\lambda}=\frac{1}{2} \lambda= \pm 1 / 2$ channel, the nucleon dispersion relation was only meaningfully satisfied up to $\simeq 1.75 \mathrm{GeV}$. Variational analyses within the phase modified distillation spaces yielded agreement with the nucleon dispersion relation in excess of $3 \mathrm{GeV}$.

The determination of several renormalized isovector charges of the nucleon was used as further evidence for the utility of merging distillation with momentum smearing. Matrix elements at rest and for $a p_{z}=(2 \pi / L)$ were computed without phasing. These were then compared to identical matrix elements computed in a boosted frame $\left[a p_{z}=4(2 \pi / L)\right]$ with and without momentum phases. Our aim was to demonstrate consistency between charges computed in different (forward) frames. This is an especially nuanced venture, as numerous form factors begin to compete as the momentum frame is varied. Furthermore, the momentum smearing procedure certainly improves overlap onto unwanted single and multiparticle excited states. A proper treatment of this consistency requires dedicated calculations of nucleon form factors at several lattice spacings/volumes and pion masses. These encouraging results nevertheless establish the feasibility of future calculational paradigms requiring distillation at high momenta. Our attention is now turned to such studies.

\section{ACKNOWLEDGMENTS}

We thank members of the HadStruc Collaboration for invaluable discussions and scrutiny. We acknowledge the facilities of the USQCD Collaboration used for this research in part, which are funded by the Office of Science of the U.S. Department of Energy. This work used the Extreme Science and Engineering Discovery Environment (XSEDE), which is supported by the National Science Foundation under Grant No. ACI1548562 [52]. We further acknowledge the Texas Advanced Computing Center (TACC) at the University of Texas at Austin for HPC resources on Frontera that have contributed greatly to the results in this work. We gratefully acknowledge computing cycles provided by facilities at William and Mary, which were provided by contributions from the National Science Foundation (MRI Grant No. PHY-1626177), and the Commonwealth of Virginia Equipment Trust Fund. The authors acknowledge William and Mary Research Computing for providing computational resources and/or technical support that have contributed to the results reported within this paper. Calculations were performed using the Chroma [53], QUDA [54,55], QDP-JIT [56], and QPhiX [57,58] 
software libraries which were developed with support from the U.S. Department of Energy, Office of Science, Office of Advanced Scientific Computing Research and Office of Nuclear Physics, Scientific Discovery through Advanced Computing (SciDAC) program. This research was also supported by the Exascale Computing Project No. (17-SC-20-SC), a collaborative effort of the U.S. Department of Energy Office of Science and the National Nuclear Security Administration. This material is based upon work supported by the U.S. Department of Energy, Office of Science, Office of Nuclear Physics under Contract No. DE-AC05-06OR23177. C. E. is supported in part by the U.S. Department of Energy under Contract No. DEFG02-04ER41302, a Department of Energy Office of Science Graduate Student Research fellowship, through the U.S. Department of Energy, Office of Science, Office of Workforce Development for Teachers and Scientists, Office of Science Graduate Student Research (SCGSR) program, and a Jefferson Science Associates graduate fellowship. The SCGSR program is administered by the Oak Ridge Institute for Science and Education (ORISE) for the DOE. ORISE is managed by ORAU under Contract No. DESC0014664. K. O. was supported in part by U.S. DOE Grant No. DE-FG02-04ER41302 and in part by the Center for Nuclear Femtography Grants No. C2-2020-FEMT-006 and No. C2019-FEMT-002-05.

\section{APPENDIX: VARIATION OF DISTILLATION SPACE RANK}

Variability of extracted observables with $R_{\mathcal{D}}$ is a central concern of any distillation based program. Ideally $R_{\mathcal{D}}$ is chosen to sufficiently capture the spatial structure of a desired hadronic state and its neighboring excited states, while minimizing the computational cost. As was found in $[31,38,59]$, baryonic ground-state energies are reliably sampled for a distillation rank as low as $R_{\mathcal{D}}=32$. Additional eigenvectors were required in $[31,59]$ to ensure a reasonably stable low-lying excited-state spectrum. These studies were concerned with the baryon spectrum at rest; our ability to resolve the ground-state energy for $R_{\mathcal{D}}>32$ is essential for this body of work, as the density of energy eigenstates grows rapidly as a hadron's momentum is increased.

In the interest of self-consistency, we justify $R_{\mathcal{D}}=64$ in this work by extracting the ground-state nucleon energies up to $a p_{z}=4 \times(2 \pi / L)$ using the spatially local $N^{2} S_{S^{2}}{ }^{\frac{1}{}}$ interpolator, for variable distillation ranks $R_{\mathcal{D}} \in\{32,48,56\}$, both without phasing and with phasing using one unit of allowed lattice momentum (5). These results are presented in Fig. 13, together with two-state fits where feasible. Ultimately we settled on $R_{\mathcal{D}}=64$, as this ensured decent statistical precision and control of excited states to facilitate a direct comparison of phased and unphased distillation.

In the case of the unphased results, the nucleon effective energy $a p_{z}=0$ is as expected stable across all ranks $R_{\mathcal{D}}$ considered, with the only discernible difference being statistical precision. Although the two-state fits to the $a p_{z}=\{1,2\} \times(2 \pi / L)$ data are consistent for each $R_{\mathcal{D}}$, the correlator signal is seen to rapidly degrade as the distillation rank is reduced from $R_{\mathcal{D}}=64$. Furthermore, the highly boosted nucleon energies are dominated by statistical fluctuations for $a p_{z} \geq 3 \times(2 \pi / L)$. These data demonstrate that $R_{\mathcal{D}}=64$ provides a computationally efficient number of distillation vectors to access baryon observables at high momentum, and that fewer eigenvectors lead to increasingly larger statistical uncertainties.

An important finding of this eigenvector study is that the union of momentum smearing and distillation reduces the number of eigenvectors needed to extract an observable with a level of error commensurate to an unphased extraction with more eigenvectors. For instance, the phased effective energy data and two-state fits for $R_{\mathcal{D}}=48$ are quite similar to $R_{\mathcal{D}}=64$ without phasing. Furthermore, the use of phasing leads to a modest resolution of the nucleon effective energy up to $a p_{z}=4(2 \pi / L)$ for each of $R_{\mathcal{D}} \in\{32,48,56\}$, and even allows for a wider temporal fit range. The implications of this finding cannot be understated, as envisioned structure studies with distillation (e.g., PDFs) would be exponentially more costly were momentum smearing not utilized. 

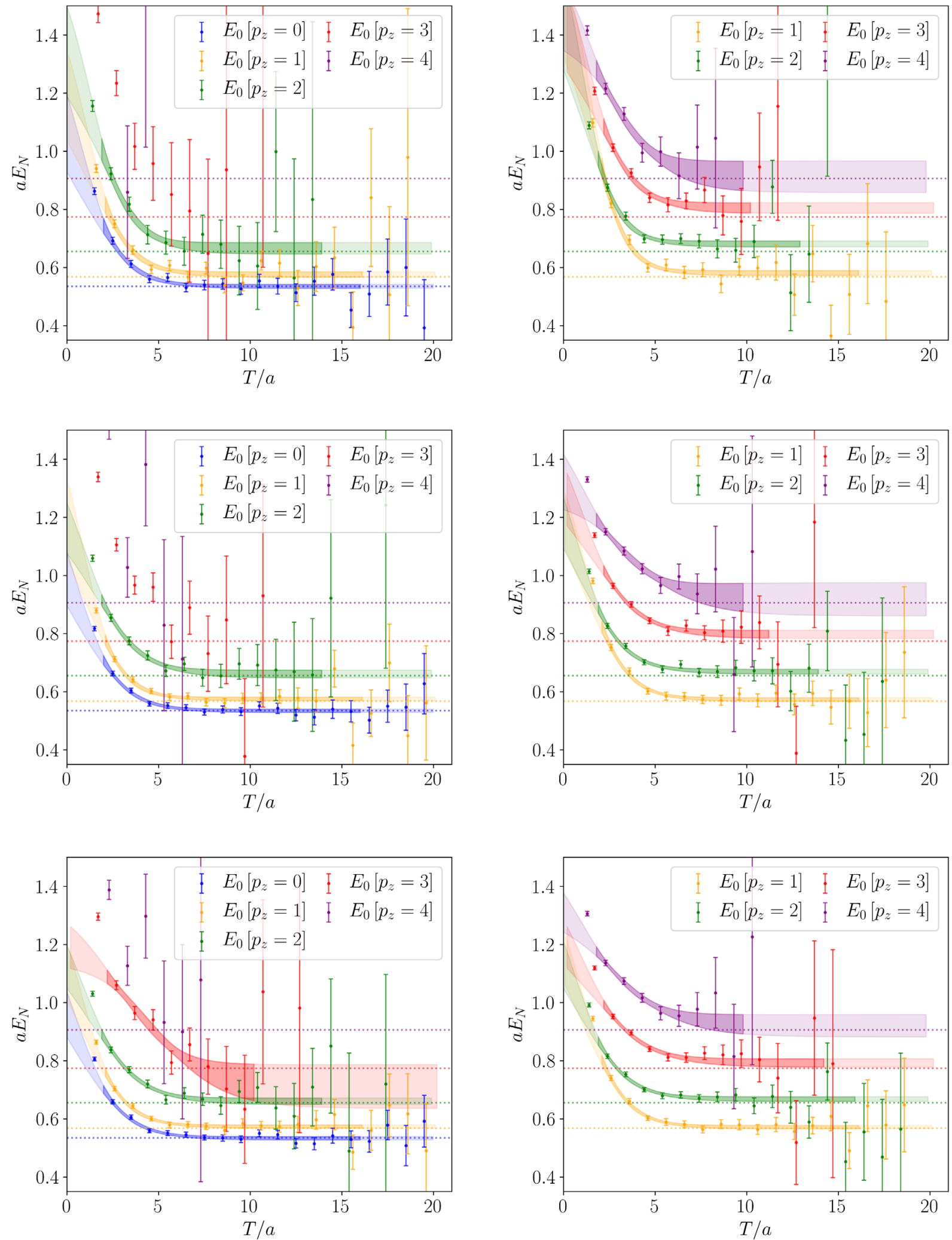

FIG. 13. Nucleon effective energies on the $a 094 m 358$ ensemble using the spatially local $N^{2} S_{S} \frac{1}{2}{ }^{+}$interpolator. The left [right] columns are from unphased [phased Eq. (5)] eigenvectors with signal-to-noise ratios $\geq 1.35[\geq 2]$. The top, middle, and bottom rows are for a distillation rank of $R_{\mathcal{D}}=\{32,48,56\}$, respectively. Two-state fits (bands) are darkened in temporal range included in the fits, while dashed lines are expectations from the continuum nucleon dispersion relation. 
[1] S. Güsken, A study of smearing techniques for hadron correlation functions, Nucl. Phys. B, Proc. Suppl. 17, 361 (1990).

[2] C. R. Allton, C. T. Sachrajda, R. M. Baxter, S. P. Booth, K. C. Bowler, S. Collins, D. S. Henty, R. D. Kenway, B. J. Pendleton, D. G. Richards, J. N. Simone, A. D. Simpson, B. E. Wilkes, and C. Michael (UKQCD Collaboration), Gauge-invariant smearing and matrix correlators using Wilson fermions at $\beta=6.2$, Phys. Rev. D 47, 5128 (1993).

[3] G. S. Bali, B. Lang, B. U. Musch, and A. Schäfer, Novel quark smearing for hadrons with high momenta in lattice QCD, Phys. Rev. D 93, 094515 (2016).

[4] C. Kallidonis, S. Syritsyn, M. Engelhardt, J. Green, S. Meinel, J. Negele, and A. Pochinsky, Nucleon electromagnetic form factors at high $Q^{2}$ from Wilson-clover fermions, Proc. Sci., LATTICE2018 (2018) 125 [arXiv:1810.04294].

[5] G. S. Bali, S. Collins, M. Göckeler, R. Rödl, A. Schäfer, and A. Sternbeck, Nucleon generalized form factors from twoflavor lattice QCD, Phys. Rev. D 100, 014507 (2019).

[6] F. Bahr, D. Banerjee, F. Bernardoni, M. Koren, H. Simma, and R. Sommer, Extraction of bare form factors for $B_{s} \rightarrow$ $K \ell \nu$ decays in nonperturbative HQET, Int. J. Mod. Phys. A 34, 1950166 (2019).

[7] A. Bazavov et al. (Fermilab Lattice and MILC Collaborations), $B_{s} \rightarrow K \ell \nu$ decay from lattice QCD, Phys. Rev. D 100, 034501 (2019).

[8] X. Ji, Parton Physics on a Euclidean Lattice, Phys. Rev. Lett. 110, 262002 (2013).

[9] X. Ji, Parton physics from large-momentum effective field theory, Sci. China Phys. Mech. Astron. 57, 1407 (2014).

[10] H.-W. Lin, J.-W. Chen, X. Ji, L. Jin, R. Li, Y.-S. Liu, Y.-B. Yang, J.-H. Zhang, and Y. Zhao (LP ${ }^{3}$ Collaboration), Proton Isovector Helicity Distribution on the Lattice at Physical Pion Mass, Phys. Rev. Lett. 121, 242003 (2018).

[11] C. Alexandrou, K. Cichy, M. Constantinou, K. Jansen, A. Scapellato, and F. Steffens, Transversity parton distribution functions from lattice QCD, Phys. Rev. D 98, 091503 (2018).

[12] J.-W. Chen, L. Jin, H.-W. Lin, Y.-S. Liu, Y.-B. Yang, J.-H. Zhang, and Y. Zhao, Lattice calculation of parton distribution function from LaMET at physical pion mass with large nucleon momentum, arXiv:1803.04393.

[13] C. Alexandrou, K. Cichy, M. Constantinou, K. Jansen, A. Scapellato, and F. Steffens, Light-Cone Parton Distribution Functions from Lattice QCD, Phys. Rev. Lett. 121, 112001 (2018).

[14] Z.-Y. Fan, Y.-B. Yang, A. Anthony, H.-W. Lin, and K.-F. Liu, Gluon Quasi-Parton-Distribution Functions from Lattice QCD, Phys. Rev. Lett. 121, 242001 (2018).

[15] T. Izubuchi, L. Jin, C. Kallidonis, N. Karthik, S. Mukherjee, P. Petreczky, C. Shugert, and S. Syritsyn, Valence parton distribution function of pion from fine lattice, Phys. Rev. D 100, 034516 (2019).

[16] X. Gao, L. Jin, C. Kallidonis, N. Karthik, S. Mukherjee, P. Petreczky, C. Shugert, S. Syritsyn, and Y. Zhao, Valence parton distribution of the pion from lattice QCD: Approaching the continuum limit, Phys. Rev. D 102, 094513 (2020).

[17] C. Alexandrou, K. Cichy, M. Constantinou, K. Hadjiyiannakou, K. Jansen, A. Scapellato, and F. Steffens (Extended Twisted Mass Collaboration), Unpolarized and Helicity Generalized
Parton Distributions of the Proton within Lattice QCD, Phys. Rev. Lett. 125, 262001 (2020).

[18] G. S. Bali, V. M. Braun, S. Bürger, M. Göckeler, M. Gruber, F. Hutzler, P. Korcyl, A. Schäfer, A. Sternbeck, and P. Wein (RQCD Collaboration), Light-cone distribution amplitudes of pseudoscalar mesons from lattice QCD, J. High Energy Phys. 08 (2019) 065.

[19] G. S. Bali, V. M. Braun, M. Göckeler, M. Gruber, F. Hutzler, P. Korcyl, A. Schäfer, and P. Wein, Pion and Kaon Distribution Amplitudes from lattice QCD: towards the continuum limit, Proc. Sci., LATTICE2018 (2018) 107 [arXiv:1811.06050].

[20] G. S. Bali, V. M. Braun, B. Gläßle, M. Göckeler, M. Gruber, F. Hutzler, P. Korcyl, A. Schäfer, P. Wein, and J.-H. Zhang, Pion distribution amplitude from Euclidean correlation functions: Exploring universality and higher-twist effects, Phys. Rev. D 98, 094507 (2018).

[21] B. Joó, J. Karpie, K. Orginos, A. V. Radyushkin, D. G. Richards, R. S. Sufian, and S. Zafeiropoulos, Pion valence structure from Ioffe-time parton pseudodistribution functions, Phys. Rev. D 100, 114512 (2019).

[22] B. Joó, J. Karpie, K. Orginos, A. Radyushkin, D. Richards, and S. Zafeiropoulos, Parton distribution functions from Ioffe time pseudo-distributions, J. High Energy Phys. 12 (2019) 081.

[23] R. S. Sufian, J. Karpie, C. Egerer, K. Orginos, J.-W. Qiu, and D. G. Richards, Pion valence quark distribution from matrix element calculated in lattice QCD, Phys. Rev. D 99, 074507 (2019).

[24] B. Joó, J. Karpie, K. Orginos, A. V. Radyushkin, D. G. Richards, and S. Zafeiropoulos, Parton Distribution Functions from Ioffe Time Pseudodistributions from Lattice Calculations: Approaching the Physical Point, Phys. Rev. Lett. 125, 232003 (2020).

[25] Z. Fan, R. Zhang, and H.-W. Lin, Nucleon gluon distribution function from 2+1+1-flavor lattice QCD, arXiv:2007.16113.

[26] A. V. Radyushkin, Quasi-parton distribution functions, momentum distributions, and pseudo-parton distribution functions, Phys. Rev. D 96, 034025 (2017).

[27] Y.-Q. Ma and J.-W. Qiu, Extracting parton distribution functions from lattice QCD calculations, Phys. Rev. D 98, 074021 (2018).

[28] Y.-Q. Ma and J.-W. Qiu, Exploring Partonic Structure of Hadrons Using ab initio Lattice QCD Calculations, Phys. Rev. Lett. 120, 022003 (2018).

[29] R. S. Sufian, C. Egerer, J. Karpie, R. G. Edwards, B. Joó, Y.-Q. Ma, K. Orginos, J.-W. Qiu, and D. G. Richards, Pion valence quark distribution from current-current correlation in lattice QCD, Phys. Rev. D 102, 054508 (2020).

[30] M. Peardon, J. Bulava, J. Foley, C. Morningstar, J. Dudek, R. G. Edwards, B. Joó, H.-W. Lin, D. G. Richards, and K. J. Juge (Hadron Spectrum Collaboration), Novel quark-field creation operator construction for hadronic physics in lattice QCD, Phys. Rev. D 80, 054506 (2009).

[31] R. G. Edwards, J. J. Dudek, D. G. Richards, and S. J. Wallace, Excited state baryon spectroscopy from lattice QCD, Phys. Rev. D 84, 074508 (2011).

[32] J. J. Dudek and R. G. Edwards, Hybrid baryons in QCD, Phys. Rev. D 85, 054016 (2012). 
[33] J. J. Dudek, R. G. Edwards, M. J. Peardon, D. G. Richards, and C. E. Thomas (Hadron Spectrum Collaboration), Highly Excited and Exotic Meson Spectrum from Dynamical Lattice QCD, Phys. Rev. Lett. 103, 262001 (2009).

[34] J. J. Dudek, R. G. Edwards, M. J. Peardon, D. G. Richards, and C. E. Thomas, Toward the excited meson spectrum of dynamical QCD, Phys. Rev. D 82, 034508 (2010).

[35] J. J. Dudek, R. G. Edwards, B. Joó, M. J. Peardon, D. G. Richards, and C. E. Thomas (Hadron Spectrum Collaboration), Isoscalar meson spectroscopy from lattice QCD, Phys. Rev. D 83, 111502 (2011).

[36] L. Liu, G. Moir, M. Peardon, S. M. Ryan, C. E. Thomas, P. Vilaseca, J. J. Dudek, R. G. Edwards, B. Joo, and D. G. Richards (Hadron Spectrum Collaboration), Excited and exotic charmonium spectroscopy from lattice QCD, J. High Energy Phys. 07 (2012) 126.

[37] J. J. Dudek, R. G. Edwards, P. Guo, and C. E. Thomas (Hadron Spectrum Collaboration), Toward the excited isoscalar meson spectrum from lattice QCD, Phys. Rev. D 88, 094505 (2013).

[38] C. Egerer, D. Richards, and F. Winter, Controlling excitedstate contributions with distillation in lattice QCD calculations of nucleon isovector charges $g_{S}^{u-d}, g_{A}^{u-d}, g_{T}^{u-d}$, Phys. Rev. D 99, 034506 (2019).

[39] B. Yoon, R. Gupta, T. Bhattacharya, M. Engelhardt, J. Green, B. Joó, H.-W. Lin, J. Negele, K. Orginos, A. Pochinsky, D. Richards, S. Syritsyn, and F. Winter (Nucleon Matrix Elements (NME) Collaboration), Controlling excited-state contamination in nucleon matrix elements, Phys. Rev. D 93, 114506 (2016).

[40] B. Yoon, Y.-C. Jang, R. Gupta, T. Bhattacharya, J. Green, B. Joó, H.-W. Lin, K. Orginos, D. Richards, S. Syritsyn, and F. Winter (Nucleon Matrix Elements (NME) Collaboration), Isovector charges of the nucleon from 2+1-flavor QCD with clover fermions, Phys. Rev. D 95, 074508 (2017).

[41] C. Morningstar and M. J. Peardon, Analytic smearing of SU(3) link variables in lattice QCD, Phys. Rev. D 69, 054501 (2004).

[42] D. C. Moore and G. T. Fleming, Angular momentum on the lattice: The case of nonzero linear momentum, Phys. Rev. D 73, 014504 (2006); Erratum, Phys. Rev. D 74, 079905(E) (2006).

[43] C. E. Thomas, R. G. Edwards, and J. J. Dudek (Hadron Spectrum Collaboration), Helicity operators for mesons in flight on the lattice, Phys. Rev. D 85, 014507 (2012).

[44] C. C. Chang, A. N. Nicholson, E. Rinaldi, E. Berkowitz, N. Garron, D. A. Brantley, H. Monge-Camacho, C. J. Monahan, C. Bouchard, M. A. Clark, B. Joó, T. Kurth, K. Orginos, P. Vranas, and A. Walker-Loud, A per-cent-level determination of the nucleon axial coupling from quantum chromodynamics, Nature (London) 558, 91 (2018).

[45] G. S. Bali, S. Collins, B. Gläßle, M. Göckeler, J. Najjar, R. H. Rödl, A. Schäfer, R. W. Schiel, W. Söldner, and
A. Sternbeck, Nucleon isovector couplings from $N_{f}=2$ lattice QCD, Phys. Rev. D 91, 054501 (2015).

[46] R. Horsley, Y. Nakamura, A. Nobile, P. E. L. Rakow, G. Schierholz, and J. M. Zanotti, Nucleon axial charge and pion decay constant from two-flavor lattice QCD, Phys. Lett. B 732, 41 (2014).

[47] P. A. Zyla et al. (Particle Data Group), Review of particle physics, Prog. Theor. Exp. Phys. 2020, 083 C01 (2020).

[48] Y.-C. Jang, R. Gupta, B. Yoon, and T. Bhattacharya, Axial Vector Form Factors from Lattice QCD that Satisfy the PCAC Relation, Phys. Rev. Lett. 124, 072002 (2020).

[49] J. Liang, Y.-B. Yang, K.-F. Liu, A. Alexandru, T. Draper, and R. S. Sufian, Lattice calculation of nucleon isovector axial charge with improved currents, Phys. Rev. D 96, 034519 (2017).

[50] K.-I. Ishikawa, Y. Kuramashi, S. Sasaki, N. Tsukamoto, A. Ukawa, and T. Yamazaki (PACS Collaboration), Nucleon form factors on a large volume lattice near the physical point in 2+1 flavor QCD, Phys. Rev. D 98, 074510 (2018).

[51] C. Alexandrou, M. Constantinou, K. Hadjiyiannakou, K. Jansen, C. Kallidonis, G. Koutsou, and A. Vaquero AvilesCasco, Nucleon axial form factors using $N_{f}=2$ twisted mass fermions with a physical value of the pion mass, Phys. Rev. D 96, 054507 (2017).

[52] J. Towns et al., XSEDE: Accelerating scientific discovery, Comput. Sci. Eng. 16, 62 (2014).

[53] R. G. Edwards and B. Joó (SciDAC, LHPC, and UKQCD Collaborations), The chroma software system for lattice QCD, Nucl. Phys. B, Proc. Suppl. 140, 832 (2005).

[54] M. Clark, R. Babich, K. Barros, R. Brower, and C. Rebbi, Solving lattice QCD systems of equations using mixed precision solvers on GPUs, Comput. Phys. Commun. 181, 1517 (2010).

[55] R. Babich, M. A. Clark, and B. Joo, Parallelizing the QUDA library for multi-GPU calculations in lattice quantum chromodynamics, in SC 10 (Supercomputing 2010) (2010) [arXiv:1011.0024].

[56] F. Winter, M. Clark, R. Edwards, and B. Joó, A Framework for lattice QCD calculations on GPUs, in 28th IEEE International Parallel and Distributed Processing Symposium (2014) [arXiv:1408.5925].

[57] B. Joó, D. Kalamkar, K. Vaidyanathan, M. Smelyanskiy, K. Pamnany, V. Lee, P. Dubey, and W. Watson, in Supercomputing, Lecture Notes in Computer Science Vol. 7905, edited by J. Kunkel, T. Ludwig, and H. Meuer (Springer, Berlin Heidelberg, 2013), pp. 40-54.

[58] B. Joo, D. D. Kalamkar, T. Kurth, K. Vaidyanathan, and A. Walden, in Optimizing Wilson-Dirac Operator and Linear Solvers for Intel KNL 2016. ISC: High Performance Computing (2016), Vol. 30, pp. 415-427.

[59] T. Khan, D. Richards, and F. Winter, The positive-parity baryon spectrum and the role of hybrid baryons, arXiv: 2010.03052. 